\title{
Parametrics of electromagnetic searches for axion dark matter
}

\author{
Robert Lasenby $\odot^{*}$ \\ Stanford Institute for Theoretical Physics, Stanford University, Stanford, California 94305, USA
}

(Received 4 December 2020; accepted 8 March 2021; published 9 April 2021)

\begin{abstract}
Light axionlike particles occur in many theories of beyond-Standard-Model physics, and may make up some or all of the Universe's dark matter. One of the ways they can couple to the Standard Model is through the electromagnetic $F_{\mu \nu} \tilde{F}^{\mu \nu}$ portal, and there is a broad experimental program, covering many decades in mass range, aiming to search for axion dark matter via this coupling. In this paper, we derive limits on the absorbed power, and coupling sensitivity, for a broad class of such searches. We find that standard techniques, such as resonant cavities and dielectric haloscopes, can achieve $\mathcal{O}(1)$-optimal axion-massaveraged signal powers, for given volume and magnetic field. For low-mass (frequency $\ll \mathrm{GHz}$ ) axions, experiments using static background magnetic fields generally have suppressed sensitivity; we discuss the physics of this limitation, and propose experimental methods to avoid it, such as microwave up-conversion experiments. We also comment on the detection of other forms of dark matter, including dark photons, as well as the detection of relativistic hidden-sector particles.
\end{abstract}

DOI: 10.1103/PhysRevD.103.075007

\section{INTRODUCTION}

Axionlike particles, in particular the QCD axion, are a well-motivated dark matter (DM) candidate. They occur in many models of beyond-Standard-Model physics, and can naturally be light and weakly coupled, allowing them to be stable and difficult to detect. There are also a number of early-Universe production mechanisms, which can produce them in the correct abundance to be the DM [1-8].

A wide range of existing and proposed experiments aim to detect axion DM candidates. These span many decades of mass range, and target a variety of possible couplings to the Standard Model (SM) [9]. In this paper, we will focus on the $a F_{\mu \nu} \tilde{F}^{\mu \nu}$ axion-photon-photon coupling, and address the sensitivity limits on such experimentshow small a DM-SM coupling could we possibly detect, given the dimensions, timescales, sensors etc. available? We choose the $a F_{\mu \nu} \tilde{F}^{\mu \nu}$ coupling partly because, for a generic QCD axion, this coupling must lie within a fairly narrow (logarithmic) range $[4,10]$; it is also a generic feature of many other axionlike-particle models [11]. In addition, it represents a particularly easily analyzed example of the kind of sensitivity limits we are interested in.

We derive bounds on the power absorbed by axion DM experiments, under fairly general assumptions, in terms of

*rlasenby@stanford.edu

Published by the American Physical Society under the terms of the Creative Commons Attribution 4.0 International license. Further distribution of this work must maintain attribution to the author(s) and the published article's title, journal citation, and DOI. Funded by SCOAP ${ }^{3}$. the magnetic field energy maintained inside the experimental volume. We also derive related limits on the achievable sensitivity for such experiments, using the tools of quantum measurement theory. These analyses are analogous to those developed in the gravitational wave detection literature (see e.g., $[12,13]$ and references therein).

For low-mass (frequency $\ll \mathrm{GHz}$ ) axions, we review why static-background-field experiments generally have suppressed sensitivity (compared to their scaling at higher frequencies), and point out that this suppression can be alleviated in a number of ways, potentially motivating new experimental concepts. In particular, we point out that up-conversion experiments using a magnetic field oscillating at microwave frequencies can have parametrically better scaling for small axion masses, and discuss the experimental details in a companion paper [14].

Similar sensitivity analyses can be applied directly to other forms of DM that couple to the SM photon, e.g., dark photon DM with a kinetic mixing. Analogous ideas can also be applied to other kinds of DM-SM couplings, and beyond that, to the detection of general hidden-sector states. We comment on some of these extensions later in the paper, and in the Conclusions.

\section{A. Summary of results}

Here, we give a brief summary of our main results. Suppose that dark matter consists of an axionlike particle $a$ of mass $m_{a}$, with a coupling $\mathcal{L} \supset-\frac{1}{4} g_{a \gamma \gamma} a F_{\mu \nu} \tilde{F}^{\mu \nu}$ to the $\mathrm{SM}$. For nonrelativistic axion DM, this acts as an "effective current" $J^{(a)} \simeq g_{\text {ary }} \dot{a} B$, where $B$ is the magnetic field. 
Suppose that we have some laboratory system with which we will try to detect the axion DM. If we want to search for axions over a mass range $\Delta m$, then for $g_{a \gamma \gamma}$ small enough that the target system is in the linear response regime, the expected time-averaged absorbed power from the axion effective current, at the least favorable axion mass, satisfies (under assumptions that we will discuss)

$$
\bar{P} \leq \pi \frac{g_{a \gamma \gamma}^{2} \rho_{a} \bar{U}_{B}}{\Delta m}
$$

where $\rho_{a}$ is the energy density of the axion DM, and $\bar{U}_{B}$ is the time-averaged magnetic field energy in the experimental volume (ignoring magnetic fields on very small spatial scales). Here, the expectation value includes integrating over the unknown phases of the axion signal [otherwise, there could be $\mathcal{O}\left(g_{\text {ayr }}\right)$ components of the absorbed power]. This bound applies to target systems for which the imaginary part of the response function is non-negative at positive frequencies; that is, the system on average absorbs energy from the axion forcing, rather than emitting. As we discuss below, $\bar{P}$ is, in many circumstances, closely related to the detectability of an axion signal. The $\bar{P} \propto$ $1 / \Delta m$ behavior corresponds to the power vs bandwidth trade-off that is a property of many detection schemes; covering a broader axion mass range in the same integration time necessarily leads to lower average signal power.

In the $\nu_{a} \gtrsim \mathrm{GHz}$ regime [where $\nu_{a}=m_{a} /(2 \pi)$ is the frequency of the axion oscillation], cavity haloscopes (as initially proposed in [15]) such as ADMX [16,17] and HAYSTAC $[18,19]$ can attain the bound in Eq. (1) to $\mathcal{O}(1)$. At higher frequencies $\left(\nu_{a} \gtrsim 10 \mathrm{GHz}\right)$, dielectric haloscope proposals $[20,21]$ can also achieve this, taking the experimental volume to be that occupied by the dielectric layers.

However, for $m_{a} \lesssim L^{-1}$, where $L$ is the length scale of the shielded experimental volume, the electromagnetic (EM) modes at frequencies $\sim \nu_{a}$ are naturally in the quasistatic regime. In that case, a static-background-field experiment has

$$
\bar{P} \lesssim \pi \frac{g_{a \gamma \gamma}^{2} \rho_{a} \bar{U}_{B}}{\Delta m}\left(m_{a} L\right)^{2} .
$$

This suppression affects low-frequency $\left(\nu_{a} \ll \mathrm{GHz}\right)$ axion DM detection proposals such as [22], ABRACADABRA [23], and DM Radio [24]. As discussed below, the scaling of the detectability limit is similarly affected, with the minimum detectable $g_{\text {ary }}$ increased by $\sim\left(m_{a} L\right)^{-1}$. Our results agree parametrically with those of $[24,25]$, which analyze axion DM detection in the quasistatic regime, using an inductive pickup and linear phase-invariant amplification.

Even under the assumptions leading to Eq. (1), the quasistatic suppression is not inevitable. To alleviate it for static background magnetic fields, we would need to enhance the quantum fluctuations of the EM fields that couple to the axion effective current, at frequencies $\sim \nu_{a}$. One way to achieve this is for the field fluctuations to "borrow" energy from some other source, e.g., magnetization energy in a material, or a circuit component with negative differential resistance. The practicality of such concepts requires further investigation.

The quasistatic suppression can also be alleviated by performing an up-conversion experiment, in which the background magnetic field is oscillating at a frequency $\gtrsim L^{-1}$. Up-conversion experiments have been proposed in the optical range [26-28], but the relatively small amplitude of achievable optical-frequency fields means that they would have relatively poor sensitivity. Larger magnetic fields are attainable at lower frequencies; in particular, it is routine to obtain magnetic fields of $\sim 0.1 \mathrm{~T}$ at $\sim \mathrm{GHz}$ frequencies in superconducting (SRF) cavities $[29,30]$.

These field strengths were noted in [31], which proposed a SRF up-conversion experiment. ${ }^{1}$ However, they mainly considered $\nu_{a} \sim \mathrm{GHz}$, for which static-field experiments do not encounter the quasistatic suppression. Consequently, the only benefit of a SRF experiment would be the higher cavity quality factor, which is unlikely to overcome the disadvantages of smaller background magnetic field, higher temperature (due to cooling power requirements), and drive-related noise issues. However, as we point out here, for $\nu_{a} \ll \mathrm{GHz}$, the lack of quasistatic suppression may make up-conversion more competitive. We investigate this possibility in more detail in a companion paper [14] (see also [34]).

\section{Detectability}

In the above paragraphs, we discussed the average power absorbed from the axion effective current. It is obvious that, other things being equal, a higher absorbed power makes it easier to detect axion DM. However, in comparing different experiments, other things are often not equal, and more generally, it is useful to have quantitative limits on how small a coupling can be detected.

By using quantum measurement techniques [35], we could in principle detect extremely small $g_{a \gamma \gamma}$, for a given $\bar{U}_{B}$. For example, by preparing a cavity mode in a largenumber Fock state, we could Bose-enhance the absorption (and emission) of axions [36]. However, such techniques are often difficult to implement; in axion experiments, the only similar measurement demonstrated so far is HAYSTAC's squeezed state receiver, which is planned to deliver a factor 2 scan rate improvement in the forthcoming Phase II run [19].

To understand different signal detection techniques, we can consider splitting our laboratory system into a "target" part, which couples to the axion effective current, and a

\footnotetext{
${ }^{1}$ Microwave up-conversion experiments were also proposed in $[32,33]$, but their sensitivity projections rely on an alternative interpretation of axion electrodynamics; see Sec. IV D.
} 
separate "readout" part, which couples to the target. One common technique used in readout schemes is linear, phase-invariant amplification (in particular, it is employed by almost all existing and proposed axion detection experiments at microwave frequencies and below). For different experimental setups, we can place limits on the SNR obtained, in analogy to the absorbed power limit from Eq. (1).

If a linear amplifier is employed in op-amp mode [35], and is subject to the "standard quantum limit" (SQL) [37] (see Appendix A), then the SNR obtained, averaged across a fractionally small axion mass range $\Delta m$, is bounded by

$$
\overline{\mathrm{SNR}^{2}} \lesssim C \pi\left(g^{2} \rho_{a} \bar{U}_{B}\right)^{2} \frac{t Q_{a}}{\omega_{1}^{3} \gamma \Delta m}
$$

where $Q_{a} \simeq 10^{6}$ is the fractional bandwidth of the axion $\mathrm{DM}$ signal (see Sec. II D), $\omega_{1} \simeq m_{a} \pm \omega_{B}$ is the oscillation frequency of the axion effective current (assuming that the magnetic field oscillation frequency $\omega_{B}$ is narrow bandwidth), $\gamma$ is the damping rate for the target excitations (see Sec. III B), and $C$ is a constant. This expression is valid for integration times long enough to resolve all of the relevant bandwidths $\left(t>\Delta m^{-1}, Q_{a} m_{a}^{-1}, \omega_{1}^{-1}, \gamma^{-1}\right)$. Under fairly general assumptions defining $\gamma$, we show that $C \leq \pi$. Under more restricted assumptions, we show that $C \leq 3 / 2$, and that, if the target is in a thermal state at temperature $T$, then $C \leq 1 / f\left(n_{T}\right)$, where $f\left(n_{T}\right)$ is a function of the thermal occupation number at $\omega_{1}$, with $f\left(n_{T}\right) \simeq 3 / 2$ for $n_{T} \ll 1$ and $f\left(n_{T}\right) \simeq n_{T}$ for $n_{T} \gg 1 .{ }^{2} \mathrm{We}$ conjecture that $C \leq 1 / f\left(n_{T}\right)$ also holds for our more general assumptions; for details, see Sec. III B. As occurs for the average absorbed power, there is an inverse relationship between the average SNR and $\Delta m$.

Another common setup has a linear amplifier isolated from the target, e.g., using a circulator connected to a cold load $[35,38]$, to protect the target system from noise. In this case, the SNR limit is also given by Eq. (3), up to $\mathcal{O}(1)$ numerical factors. Perhaps surprisingly, in both of these cases, the improved sensitivity for $\omega_{1} \ll m_{a}$ is actually physical, and experiments using "down-conversion" in this way could theoretically achieve improved sensitivities. However, due to a number of experimental limitations, including the relatively small $\bar{U}_{B}$ values obtainable for high-frequency magnetic fields, realizing such enhancements does not seem to be practical. Additionally, if $\omega_{1} \lesssim L^{-1}$, then the EM fields are naturally in the quasistatic regime, and the SNR is suppressed by $\sim\left(\omega_{1} L\right)^{2}$, similar to the absorbed power in Eq. (2).

At higher frequencies, detectors other than amplifiers (e.g., photon counters, bolometric detectors, quasiparticle

\footnotetext{
${ }^{2}$ For up-conversion experiments, the converted power in a narrow frequency band is at most half of the value from Eq. (1), and the $\mathrm{SNR}^{2}$ value is $1 / 4$ of the value from Eq. (3).
}

detectors, etc.) become easier to implement. While we could analyze the properties of each individually, it is the case that for a wide range of setups, the sensitivity is bounded by the number of axion quanta absorbed. We can quantify this using quantum measurement theory. The fundamental quantum limit (FQL) [39-41] for signal detection is determined by the quantum fluctuations of the EM fields that couple to the axion signal. Using the arguments that lead to Eq. (1), we can constrain the frequency-integrated spectrum of these fluctuations. For general states, we cannot use this to place a bound on detectability, since there may be canceling contributions to this integral. However, if the sensor interacts with the target via a damping-type interaction, e.g., an absorptive photodetector or bolometer, then its effects are equivalent to a passive load, and the quantum fluctuations of the target EM fields are the same as in an equilibrium state. In these circumstances, the sensitivity to axion DM, over a (fractionally small) mass range $\Delta m$, is bounded (at the least favorable axion mass) by

$$
\mathbb{P}_{\text {det }} \leq \bar{N}_{a} \equiv \frac{\bar{P} t_{\text {tot }}}{\omega_{1}}
$$

where $\mathbb{P}_{\text {det }}$ is the probability of detecting the axion signal. We will refer to this limit as the PQL (passive quantum limit). It has an obvious interpretation in terms of photon counting, for schemes in which axions convert to single photons, but it also applies to other setups, e.g., where a signal consists of multiple quasiparticle excitations. Coherent-state excitations of the target's EM fields leave their quantum fluctuations unchanged, so do not affect the PQL. As in the SQL case, the $1 / \omega_{1}$ enhancement for small $\omega_{1}$ is physical, but probably not practical.

If we take $\gamma$ small enough so that the assumptions behind Eq. (3) no longer hold, the maximum SNR from a linear amplifier, isolated behind a circulator with a cold load, saturates to $\sim \bar{N}_{a}$ (as does the SQL op-amp limit). This is as we would expect, since the target fluctuations are as if we had connected a passive load.

As we emphasized above, it is certainly possible to do better than the PQL, by using techniques involving "nonclassical" EM field states. One important example is using linear amplifiers with correlated backaction and imprecision noise. By optimizing this correlation, we can in theory obtain the "quantum limit" [35,37], for which the SNR bound is

$$
\overline{\mathrm{SNR}^{2}} \lesssim \frac{\pi}{2}\left(g^{2} \rho_{a} \bar{U}_{B}\right)^{2} \frac{t Q_{a}}{\omega_{1}^{3} \gamma^{2}\left(1+n_{T}\right)^{2}}
$$

where the notation is as for Eq. (3). Unlike the SQL and PQL, this limit does not involve $\Delta m$; a QL-limited experiment is inherently broadband if we can optimize the amplifier properties across a wide bandwidth. In the 
quasistatic limit, the SNR is again suppressed by $\sim\left(\omega_{1} L\right)^{2}$. Superconducting quantum interference device (SQUID) amplifiers (as proposed e.g., [22] and the ABRACADABRA axion DM detection experiment [23]) can, in some circumstances, attain near-QL performance [42]. The fact that the QL-limited sensitivity can, in some regimes, be better than the PQL, corresponds to the amplifier backaction enhancing the quantum fluctuations of the target EM fields.

While some other measurement schemes, such as backaction evasion [35], do not have such general limits on their sensitivity, we could still analyze their performance given more specific assumptions. In this paper, we will restrict our discussion to the amplifier and PQL limits introduced above. One reason for doing so is that, taken together, they apply to almost all existing and proposed axion DM detection experiments.

As we will discuss, these limits help in understanding what can and cannot enhance an experiment's sensitivity to axion DM, and in comparing the potential sensitivity of different kinds of experiments.

\section{AXION DM INTERACTIONS}

We will suppose that dark matter consists of an axionlike particle $a$, with a coupling to the SM photon. This has Lagrangian $^{3}$

$$
\begin{aligned}
\mathcal{L} & \supset \frac{1}{2}\left(\partial_{\mu} a\right)^{2}-V(a)-\frac{1}{4} g_{a \gamma \gamma} a F_{\mu \nu} \tilde{F}^{\mu \nu} \\
& =\frac{1}{2}\left(\partial_{\mu} a\right)^{2}-V(a)+g_{a \gamma \gamma} a E \cdot B,
\end{aligned}
$$

where $V(a)$ is the potential for the axion; in general, only the mass term $V(a)=\frac{1}{2} m_{a}^{2} a^{2}$ will be important for us.

As far as is understood, almost all production mechanisms for axion DM result in the field today being in a coherent, classical-like state [1-4,43]; if the axion mass is small, $m_{a} \ll \mathrm{eV}$, then the average occupation number within the Milky Way is $\gg 1$. Since $g_{a \gamma \gamma}$ (and other couplings) are constrained to be very small, interactions with a detector will have a negligible effect on the DM's state. Consequently, for the purposes of detection, we can treat the DM oscillation as a fixed classical background field.

The $F \tilde{F}$ term is a total derivative, $F_{\mu \nu} \tilde{F}^{\mu \nu}=2 \partial_{\mu}\left(A_{\nu} \tilde{F}^{\mu \nu}\right)$, so under integration by parts, the interaction term in the Lagrangian is equivalent to

$$
-\frac{1}{4} g a F_{\mu \nu} \tilde{F}^{\mu \nu} \rightarrow \frac{1}{2} g\left(\partial_{\mu} a\right) A_{\nu} \tilde{F}^{\mu \nu}
$$

\footnotetext{
${ }^{3}$ We take the $(+---)$ signature, and use the convention $\epsilon_{0123}=-1$. Except where indicated, we use natural units with $c=\hbar=k_{B}=1$. In general, we will abbreviate $g_{a \gamma \gamma}=g$.
}

$$
\begin{aligned}
=\frac{1}{2} g(A \cdot(\dot{a} B & \left.+(\nabla a) \times E)-A_{0} \nabla a \cdot B\right) \\
= & -\frac{1}{2} A^{\mu} J_{\mu}^{(a)} .
\end{aligned}
$$

Note that, for our signature choice, the components of the usual three-vector potential, which we will denote $A$, are $-A_{i}$. To begin with, we will focus on the case of a spatially constant (zero-velocity) axion DM field, $\nabla a=0$. This is usually a good approximation, since the DM is highly nonrelativistic, with $v_{\mathrm{DM}} \sim 10^{-3}$ (we will come back to the consequences of the axion velocity distribution in Sec. II D). If $\nabla a=0$, then the interaction term is $\mathcal{L} \supset \frac{1}{2} g \dot{a} B \cdot A$.

\section{A. Response dynamics}

For many purposes, it will be convenient to work in a Hamiltonian framework. The Lagrangian density for the electromagnetic field can be written as

$$
\mathcal{L}=-\frac{1}{4} F_{\mu \nu} F^{\mu \nu}-A_{\mu} J^{\mu}+\mathcal{L}_{\text {matter }}
$$

where $J_{\mu}=J_{\mu}^{\mathrm{SM}}+J_{\mu}^{(a)} / 2$. If we work in Coulomb gauge, $\nabla \cdot A=0$, then for $J_{\mu}$ independent of $\dot{A}$ (as is the case for Dirac fermion matter, and a zero-velocity axion field), we obtain the Hamiltonian [44]

$H=\int d^{3} x\left(\frac{1}{2} \dot{A}^{2}+\frac{1}{2} B^{2}-J \cdot A+\frac{1}{2} J^{0} A^{0}\right)+H_{\text {matter }}$

where for Dirac fermion matter, $H_{\text {matter }}$ is independent of $A$. $A^{0}$ is not a physical degree of freedom, and can be expressed in terms of $J^{0}$ as $A^{0}(x, t)=\int d^{3} x^{\prime} \frac{J^{0}\left(x^{\prime}, t\right)}{4 \pi\left|x-x^{\prime}\right|}$.

To analyze the effect of the axion oscillation on the system, we can decompose the EM vector potential as $A=A_{0}+A_{1}$, where $A_{0} \equiv\langle A\rangle$ in the absence of an axion oscillation. In the notation of Appendix A, $A_{1}=\Delta A$. If we assume that $A_{1}$ is small compared to $A_{0}$, then

$$
\begin{gathered}
A \cdot B=A \cdot(\nabla \times A) \simeq A_{0} \cdot\left(\nabla \times A_{0}\right)+A_{1} \cdot\left(\nabla \times A_{0}\right) \\
+A_{0} \cdot\left(\nabla \times A_{1}\right)
\end{gathered}
$$

so the axion interaction term can be expanded as

$$
A \cdot B \simeq A_{0} \cdot B_{0}+2 A_{1} \cdot B_{0}+\nabla \cdot\left(A_{1} \times A_{0}\right) .
$$

In general, $B_{0}$ will have some time dependence. To start with, we will assume that the time dependence and spatial profile factorize, so $B_{0}=B_{0}(t) b(x)$, as is the case e.g., for a cavity standing mode (we will revisit this in Sec. II D). We 
can decompose $A_{1}=A_{b} b+A_{\perp}$, where $\int d V A_{\perp} \cdot b=0$; we will also write $A_{0}=A_{b}^{(0)} b+A_{\perp}^{(0)}$, etc. Then, writing $V_{b} \equiv \int d V b^{2}$, the $A_{b}$-dependent parts of the Hamiltonian are

$$
\begin{aligned}
H & =V_{b}\left(\dot{A}_{b}^{(0)} \dot{A}_{b}+\frac{1}{2} \dot{A}_{b}^{2}-g \dot{a} A_{b} B_{0}\right) \\
& +\int d V\left(\frac{1}{2} B^{2}-J_{\mathrm{SM}} \cdot A\right)+\ldots
\end{aligned}
$$

The first two terms are the only ones depending on $\dot{A}_{b}$. The conjugate momentum to $A$ is $E$, so the conjugate momentum to $A_{b}$ is $-V_{b} E_{b}=V_{b} \dot{A}_{b}$ [since $\int d V\left(\nabla A_{0}\right)$. $\left.b=-\int d V A_{0} \nabla \cdot b=0\right]$, with equal-time commutation relation $\left[\hat{A}_{b}, \hat{E}_{b}\right]=-i / V_{b}$. Consequently, the Hamiltonian for $A_{b}$ is analogous to that for a $1 \mathrm{D}$ oscillator,

$$
\hat{H}_{1 \mathrm{D}}=\frac{\left(\hat{p}+p_{0}\right)^{2}}{2 M}-g j(t) \hat{x}+V_{\mathrm{int}}(\hat{x}, \ldots)
$$

where $\hat{x} \equiv A_{b}, \hat{p} \equiv-E_{b} V_{b}, p_{0} \equiv-E_{b}^{(0)} V_{b}, j(t) \equiv \dot{a}(t) \times$ $B_{0}(t) V_{b}$, and $M \equiv V_{b}$. Here, $V_{\text {int }}(\hat{x})$ summarizes the other terms in Eq. (16), which do not depend on $\hat{p}$.

If we consider a very short $j(t)$ pulse, turning on and off much faster than the system's dynamics, then its effect is to impulsively change $p$ by $g \int d t j(t) \equiv g J$. Averaging over possible signs of the pulse, the expected energy absorbed is $\langle W\rangle \simeq(\Delta p)^{2} /(2 M)$. In our case, since $j$ depends on the time derivative of $a$, a delta-function $j$ pulse corresponds to a step function in $a(t)$, and we have $\langle W\rangle \simeq \frac{1}{2} g^{2}(\Delta a)^{2} V_{b} B_{0}^{2}$.

This argument tells us the expected energy absorbed by the target from a very fast axion field "pulse." However, as discussed above, we expect axion DM to be a narrowbandwidth oscillation, with fractional bandwidth $\sim 10^{-6}$. If $g$ is small enough that the target is in the linear response regime, then the energy it absorbs from a finite-time $j_{t}(t)$ signal is

$$
\langle W\rangle=\frac{g^{2}}{2 \pi} \int_{-\infty}^{\infty} d \omega \omega\left|\tilde{j}_{t}(\omega)\right|^{2} \operatorname{Im} \tilde{\chi}(\omega)
$$

where $\chi$ is the linear response function for $x$ (if the dynamics are nonstationary in time, we can consider averaging over all possible starting times). A delta-function pulse has equal power at all frequencies, so

$$
\langle W\rangle=\frac{g^{2}}{2 \pi} J^{2} \int_{-\infty}^{\infty} d \omega \omega \operatorname{Im} \tilde{\chi}(\omega) .
$$

Equating this to the energy $g^{2} J^{2} /(2 M)$ absorbed from the pulse, we have

$$
\int_{-\infty}^{\infty} d \omega \omega \operatorname{Im} \tilde{\chi}(\omega)=\frac{\pi}{V_{b}}
$$

This "sum rule" is analogous to the Thomas-Reiche-Kuhn sum rule for "oscillator strengths" in atomic physics [45].

By itself, Eq. (20) does give us any limit on the response in a specific frequency range, since the integrand could have large canceling components. However, if $\omega \operatorname{Im} \tilde{\chi}(\omega)$ is always $\geq 0$, then we can bound the absorbed power from any signal. This obviously applies if the target is in its ground state (since a forcing can only add energy), or if $\operatorname{Im} \tilde{\chi}$ is equivalent to its ground-state form. For a purely harmonic oscillator, the latter is true in any state. More generally, if the target is in a mixed state, where the probability of a microstate decreases with increasing energy, then the condition also holds, as we show below.

These arguments are a generalization of the pulseabsorption argument from [21], which was used to the determine the axion-mass-averaged signal power from a dielectric haloscope.

The sum rule in Eq. (20) depends on the fact that laboratory materials are composed of particles carrying electric charges. If particles carrying magnetic charge existed (or more generally, particles with Amperian electric dipole moments), then it would be possible to violate the sum rule. This can be seen using an electricmagnetic duality transformation, under which a paramagnetic material is transformed to a material with negative electrical susceptibility. As we go over below, a dielectric medium gives $\int d \omega \omega \operatorname{Im} \tilde{\chi}(\omega)=\frac{\pi}{\epsilon V_{b}}$, so if $\epsilon<1$ over some order-one frequency range, the sum rule can be violated. This illustrates that the sum rule does have physical content, but is satisfied very generally for realistic laboratory systems.

\section{B. Fluctuation sum rules}

To apply the FQL detectability limits discussed in Appendix A, we need to understand the fluctuation spectrum of $A_{b}$. We can relate this to the response function via the Kubo formula [46], $\operatorname{Im} \tilde{\chi}(\omega)=\frac{1}{2}\left(S_{A_{b} A_{b}}(\omega)-\right.$ $S_{A_{b} A_{b}}(-\omega)$ ), where $S_{A_{b} A_{b}}(\omega)$ is the spectral density of $A_{b}$ fluctuations. Thus, the sum rule in Eq. (20) implies a corresponding sum rule for $S_{A_{b} A_{b}}$,

$$
\int_{-\infty}^{\infty} d \omega \omega S_{A_{b} A_{b}}(\omega)=\frac{\pi}{V_{b}} .
$$

We can also derive this sum rule directly from the commutation relations of the EM fields. The spectral density of $A_{b}$ fluctuations (assuming that they are stationary in time) is 
$S_{A_{b} A_{b}}(\omega) \equiv \int_{-\infty}^{\infty} d t e^{i \omega t}\left\langle\hat{A}_{b}(t) \hat{A}_{b}(0)=\mathcal{F}\left\langle\hat{A}_{b}(t) \hat{A}_{b}(0)\right\rangle\right.$

where $\hat{A}_{b}(t)$ is the Heisenberg picture operator for the system, in the absence of axion interactions (going forward, we will drop the hats). Integrating this over $\omega$,

$$
\begin{gathered}
\int_{-\infty}^{\infty} d \omega \omega S_{A_{b} A_{b}}(\omega)=\int_{-\infty}^{\infty} d \omega \omega \mathcal{F}\left\langle A_{b}(t) A_{b}(0)\right\rangle \\
=i \int_{-\infty}^{\infty} d \omega \mathcal{F}\left\langle\dot{A_{b}}(t) A_{b}(0)\right\rangle .
\end{gathered}
$$

We have

$$
E_{b}=\frac{1}{V_{b}} \int d V E \cdot b=\frac{1}{V_{b}} \int d V\left(-\nabla A_{0}-\dot{A}\right) \cdot b=-\dot{A}_{b}
$$

since $\nabla \cdot b=0$, so

$$
\int_{-\infty}^{\infty} d \omega \omega S_{A_{b} A_{b}}(\omega)=-2 \pi i\left\langle E_{b}(0) A_{b}(0)\right\rangle .
$$

If the fluctuations of $A_{b}$ are stationary in time (as e.g., a coherent state), then $\mathcal{F}\left\langle A_{b}(t) A_{b}(0)\right\rangle$ is real. So, $\left\langle A_{b}(0) E_{b}(0)\right\rangle$ is imaginary, and consequently, for equal times,

$$
\left\langle E_{b} A_{b}\right\rangle=\frac{1}{2}\left\langle\left[E_{b}, A_{b}\right]\right\rangle=\frac{i}{2 V_{b}} .
$$

Inserting this into Eq. (26) reproduces Eq. (21).

As per the previous section, we are usually interested in the fluctuations across some narrow frequency range. In general, there can be contributions to Eq. (21) from positive and negative $\omega$, leading to cancellations. However, for the ground state of the system, $S_{A_{b} A_{b}}(\omega)=0$ for $\omega<0$; for any operator $\hat{F}$,

$$
\langle 0|\hat{F}(t) \hat{F}(0)| 0\rangle=\sum_{n} e^{-i\left(\omega_{n}-\omega_{0}\right) t}|\langle n|\hat{F}| 0\rangle|^{2} .
$$

So, for the ground state, we obtain the sum rule

$$
\int_{0}^{\infty} d \omega \omega S_{A_{b} A_{b}}(\omega)=\frac{\pi}{V_{b}} .
$$

The same is true for coherent states, since their fluctuations on top of the c-number expectation value are the same as for the ground state.

If we consider a probability mixture of energy eigenstates, $\rho=\sum_{n} p_{n}|n\rangle\langle n|$, then

$$
\begin{aligned}
\langle\hat{F}(t) \hat{F}(0)\rangle \\
=\sum_{m} p_{m} \sum_{n} e^{-i\left(\omega_{n}-\omega_{m}\right) t}|\langle n|\hat{F}| m\rangle|^{2} \\
=\sum_{m} p_{m}|\langle m|\hat{F}| m\rangle|^{2}+\sum_{m} \sum_{n>m}|\langle n|\hat{F}| m\rangle|^{2} \\
\quad \times\left(p_{m} e^{-i\left(\omega_{n}-\omega_{m}\right) t}+p_{n} e^{-i\left(\omega_{m}-\omega_{n}\right) t}\right) \\
=\sum_{m} p_{m}|\langle m|\hat{F}| m\rangle|^{2}+\sum_{m} \sum_{n>m}|\langle n|\hat{F}| m\rangle|^{2} \\
\quad \times\left(\left(p_{m}-p_{n}\right) e^{-i\left(\omega_{n}-\omega_{m}\right) t}+2 p_{n} \cos \left(\left(\omega_{m}-\omega_{n}\right) t\right)\right) .
\end{aligned}
$$

Consequently, if $p_{m} \geq p_{n}$ for $m<n$, then since the odd part of this expression determines $\operatorname{Im} \tilde{\chi}(\omega)$, we have $\operatorname{Im} \tilde{\chi}(\omega) \geq 0$ for $\omega \geq 0$. Thus, for mixed states such as thermal states, the sum rule from Eq. (20) applies.

While we have focused on the fluctuations of $A_{b}$, the full interaction operator with the axion field is $-\frac{1}{2} g \dot{a} A \cdot B$ [Eq. (11)]. In most of this paper, we assume that there is a large "background" field $B_{0}$, which is basically classical, in the sense that the fluctuations around its expectation value are fractionally small. Then, as derived in Sec. II A, the interaction operator is approximately $-g \dot{a} B_{0} A_{b}$, so the fluctuations of $A_{b}$ determine the FQL for detecting an axion forcing.

\section{Effective Hamiltonians}

The above derivations relied on the conjugate momentum of $A_{i}$ being $E_{i}$; i.e., there being no other terms in the Hamiltonian involving $\dot{A}_{i}$. For example, if we were considering a dielectric medium, where the energy density is $\epsilon E^{2}$, then the conjugate momentum to $A_{i}$ would be $\epsilon E_{i}$, and we would have $\int d \omega \omega S(\omega)=\frac{\pi}{\epsilon V_{b}}$. Thus, if e.g., a resonant cavity is filled with dielectric material, the power it is able to absorb decreases [25]. From above, we know that once all of the dynamics are taken in account, $\int_{-\infty}^{\infty} d \omega \omega S(\omega)=\frac{\pi}{V_{b}}$. This implies that the "extra" fluctuations must be at frequencies above the validity of the effective Hamiltonian.

Similarly, the $\pm \infty$ limits of the $\omega$ integrals above should not be taken literally; at the very least, electroweak physics arises at some energy scale. What we can infer is that, for frequency ranges over which our description of the system is good, $\int d \omega \omega \operatorname{Im} \tilde{\chi} \leq \frac{\pi}{V_{b}}$ in the ground state, and so on.

\section{Axion velocity}

So far, we have taken the axion velocity to be zero. This will not be strictly true; in most particle physics models, axion DM in the Galaxy is expected to have a virialized velocity distribution, with typical velocity $\sim 10^{-3}$ [47]. There may also be components with smaller velocity dispersions arising from either nonvirialized "streams" of 
dark matter particles [48-51], or from bound "minihalos" (see e.g., [52,53]). In most of our discussions, we will assume that the DM velocity distribution is characterized by a single velocity dispersion scale, which is taken to be the virial velocity spread. Extending to more complicated velocity distributions is straightforward. ${ }^{4}$

As per Eq. (9), the interaction term is $\mathcal{L} \supset-\frac{1}{2} A^{\mu} J_{\mu}^{(a)}$, where

$$
J_{(a)}^{\mu}=g\left(\begin{array}{c}
(\nabla a) \cdot B \\
\dot{a} B+(\nabla a) \times E
\end{array}\right) .
$$

Compared to the zero-velocity case, the axion velocity term $\nabla a$ results in a coupling to the scalar potential $A_{0}$, as well as the vector potential $A$. However, we can work in a gauge in which $A_{0}=0$, in which case the extra coupling term is

$$
\mathcal{L} \supset A_{1} \cdot\left((\nabla a) \times E_{0}\right)
$$

(after integration by parts). For an axion wave of definite momentum, this corresponds to the $B$ field in the axion rest frame, as expected.

Consequently, we can replace $\dot{a} B_{0}$ by $\dot{a} B_{0}+(\nabla a) \times E_{0}$ as our forcing term. Since $|\nabla a| \sim v_{a}|\dot{a}| \sim 10^{-3}|\dot{a}|$, and the attainable (static) magnetic fields in laboratories are significantly larger than attainable electric fields, the $\dot{a} B_{0}$ term dominates in almost all circumstances of interest.

A more important effect of the axion velocity distribution is that the axion signal is no longer a spatially uniform, single-frequency oscillation. If the experimental volume is significantly smaller than the axion coherence length $\left[L_{\mathrm{coh}} \sim\left(v_{a} m_{a}\right)^{-1} \sim 10^{3} m_{a}^{-1}\right]$, then the axion field inside the volume is approximately uniform, but is incoherent over times $\gtrsim v_{a}^{-2} m_{a}^{-1}$, corresponding to a frequency spread $\sim v_{a}^{2} \nu_{a} \sim 10^{-6} \nu_{a}$ [where $\nu_{a} \equiv m_{a} /(2 \pi)$ is the axion frequency]. If the experimental volume is larger, then the axion field is incoherent over distances $\sim v_{a}^{-1} m_{a}^{-1}$.

We can treat the spatial variation of the axion field, as well as any time dependence of the $B_{0}$ spatial profile, by decomposing $\dot{a}(t) B_{0}(t)$ into spatially orthogonal modes, each with their own time dependence. Writing

$$
\dot{a}(t) B_{0}(t)=\sum_{i} \dot{a}_{b_{i}}(t) B_{b_{i}}(t) b_{i}
$$

where $\int d V b_{i} \cdot b_{j}=\delta_{i j} V_{b_{i}}$, and $a_{b_{i}}$ and $B_{b_{i}}$ are functions only of time, we have

$$
H_{\mathrm{int}}=g \sum_{i} \dot{a}_{b_{i}} B_{b_{i}} A_{b_{i}} .
$$

\footnotetext{
${ }^{4}$ Sufficiently small minihalos can also transit the detector on short timescales, resulting in a strongly time-dependent axion DM density [53]; we defer discussion of this case to future work.
}

We have equal-time commutation relations

$$
\left[A_{b_{i}}, E_{b_{j}}\right]=-\delta_{i j} \frac{i}{V_{b_{i}}} .
$$

So, the cross terms in the sum rule [Eq. (29)] vanish, giving

$$
\int_{-\infty}^{\infty} d \omega \omega \mathcal{F}\left\langle A_{b_{i}}\left(t_{0}\right) A_{b_{j}}\left(t_{0}+t\right)\right\rangle=\delta_{i j} \frac{\pi}{V_{b_{i}}} .
$$

This is as we would expect from the impulse argument above; over very short timescales, there is no dynamics coupling the spatially orthogonal target modes, so they have independent responses to pulses. We will discuss some of the consequences of this in Sec. III B.

\section{PARAMETRICS OF DM DETECTION}

The sum rules [Eqs. (20), (29), and (36)] derived in Sec. II can be used to bound the average power absorbed from the axion effective current, in an axion DM detection experiment. ${ }^{5}$ The simplest case is when the $B_{0}$ field is static. Over sufficiently long integration times, so that we resolve the spectral features of the axion signal, the expected time-averaged power absorbed for an axion of mass $m$ is

$$
P_{m} \simeq \frac{g^{2} B_{0}^{2} V_{b}^{2}}{2 \pi} \int_{-\infty}^{\infty} d \omega \omega S_{\dot{a} \dot{a}}(\omega) \tilde{\chi}_{i}(\omega)
$$

where we write $\tilde{\chi}_{i} \equiv \operatorname{Im} \tilde{\chi}{ }^{6}$ Averaging this over different axion masses $m$, we can use the fact that, since the axion bandwidth is small, $\delta \omega_{a} \sim 10^{-6} \mathrm{~m}$, integrating over $m$ for fixed $\omega$ is approximately the same as integrating over $\omega$ for fixed $m$,

$$
\left.\int_{0}^{\infty} d m S_{\dot{a} \dot{a}}(\omega) \simeq \int_{0}^{\infty} d \omega^{\prime} S_{\dot{a} \dot{a}}\left(\omega^{\prime}\right)\right|_{m=\omega} \simeq \pi \rho_{a} .
$$

Hence,

$$
\begin{aligned}
\int_{0}^{\infty} d m P_{m} & \simeq \frac{g^{2} B_{0}^{2} V_{b}^{2} \rho_{a}}{2} \int_{-\infty}^{\infty} d \omega \omega \tilde{\chi}_{i}(\omega) \\
& =g^{2} \rho_{a} V_{b} B_{0}^{2} \frac{\pi}{2}
\end{aligned}
$$

\footnotetext{
${ }^{5}$ In most of this paper, we view the magnetic field $B_{0}$ as a classical background field, and consider at the energy absorbed from the axion effective current $J^{(a)}=g \dot{a} B_{0}$. For a static $B_{0}$, this is equivalent to the energy absorbed from the axion field by the SM system. For time-dependent $B_{0}$, the energy absorbed "from $J^{(a)}$ " by the rest of the SM system includes energy transferred from the magnetic field. While we could always work with the energy absorbed from the axion field itself, the detectability of small signals is more closely related to the energy absorbed from $J^{(a)}$.

${ }^{6}$ For shorter integration times $t_{\text {int }}, S_{\dot{a} \dot{a}}$ should be convolved with a kernel of width $\sim 1 / t_{\text {int }}$.
} 
Consequently, the absorbed power integrated over all axion masses is set by the magnetic field energy in the $B_{0}$ field (ignoring magnetic fields on very small spatial scales; see Appendix B).

If we are interested in looking for an axion within a specific mass range $\Delta m$, then we can average $P_{m}$ over that mass range,

$$
\bar{P} \equiv \frac{1}{\Delta m} \int_{\Delta m} d m P_{m} \lesssim \frac{g^{2} \rho_{a} V_{b} B_{0}^{2}}{\Delta m} \frac{\pi}{2}
$$

where the inequality assumes that $\omega \tilde{\chi}_{i}(\omega) \geq 0$ for all $\omega$. Equality can be obtained if the response function is concentrated into the $\Delta m$ range (we will discuss some of the experimental practicalities of this in Sec. IV). Since $\min _{m \in \Delta m} P_{m} \leq \bar{P}$, the smallest absorbed power for any axion mass within the range $\Delta m$ is upper-bounded by Eq. (41). As expected, searching over a smaller axion mass range permits higher conversion powers within that range.

Equation (41) applies if $\tilde{\chi}_{i}(\omega) \geq 0$ for all $\omega>0$. Even if this is not the case, it can also apply if $\tilde{\chi}_{i}(\omega)=\tilde{\chi}_{i}{ }^{\prime}(\omega)$ for $\omega$ in the mass range $\Delta m$, where $\tilde{\chi}_{i}^{\prime}$ is the response function for a system which does have $\tilde{\chi}_{i}(\omega) \geq 0$ for all $\omega>0$. Similarly, if $\tilde{\chi}_{i} \leq C \tilde{\chi}_{i}^{\prime}$ in the relevant frequency range, for some constant $C$, then the bound in Eq. (41) should be multiplied by $C$. For example, if a system has $\tilde{\chi}_{i}(\omega)<0$ at high frequencies, but has simple low-frequency behavior, these considerations can be useful.

In many cases, instead of operating a single experimental configuration for the whole observation time, we "tune" our experiment by operating it in different configurations, one after the other. The average power for a given axion mass is the appropriately weighted sum of the powers from the different configurations, and the corresponding limits apply.

The equations above apply to the whole experimental apparatus. However, a common experimental setup is to have a conductive shield (e.g., an EM cavity) inside a larger magnetic field. If the relevant dynamics inside and outside the cavity are independent, then we can apply the above arguments to the volume inside the cavity, replacing the total magnetic field energy by the energy inside the cavity.

\section{A. PQL limits}

From Appendix A, if the quantum fluctuations of $A_{b}$ are stationary, then the $\mathcal{O}\left(g^{2}\right)$ formula for the probability of the axion interaction changing the state of the target system is

$$
\mathbb{P}_{\mathrm{ex}} \simeq \frac{g^{2} B_{0}^{2} V_{b}^{2} t_{\mathrm{exp}}}{\pi} \int_{0}^{\infty} d \omega S_{\dot{a} \dot{a}}(\omega) \bar{S}_{A_{b} A_{b}}(\omega)
$$

where $\bar{S}$ denotes the symmetrized spectral density, and we assume that $t_{\exp }$ is much longer than the inverse bandwidth of spectral features. If the fluctuations are equal to those in the ground state, then $\bar{S}_{A_{b} A_{b}}(\omega)=\tilde{\chi}_{i}(\omega)$ for $\omega>0$. In that case,

$$
\mathbb{P}_{\mathrm{ex}} \simeq \frac{g^{2} B_{0}^{2} V_{b}^{2} t_{\mathrm{exp}}}{\pi} \int_{0}^{\infty} d \omega S_{\dot{a} \dot{a}}(\omega) \tilde{\chi}_{i}(\omega) \simeq \frac{P_{m}}{m}
$$

where the latter equality holds since $S_{\dot{a} a}$ is tightly concentrated around $m$. Hence, $\mathbb{P}_{\mathrm{ex}} \simeq N_{a}$, the expected number of quanta absorbed. Since $\tilde{\chi}_{i}$ for the ground state satisfies $\tilde{\chi}_{i}(\omega) \geq 0$ for $\omega>0$, Eq. (41) holds, and so the axionmass-averaged excitation probability satisfies

$$
\overline{\mathbb{P}}_{\text {ex }} \lesssim \frac{g^{2} \rho_{a} V_{b} B_{0}^{2} t_{\text {exp }}}{m \Delta m} \frac{\pi}{2} \equiv \bar{N}_{a}
$$

where the average is taken over a fractionally small axion mass range $\Delta m$ centered on $m$. To be confident of identifying or excluding an axion signal, we need $\bar{N}_{a} \gtrsim$ few. More generally, if $\bar{S}_{A_{b} A_{b}}$ for the operational state of the detector satisfies

$$
\int_{\Delta m} d \omega \omega \bar{S}_{A_{b} A_{b}} \lesssim \frac{\pi}{2 V_{b}}
$$

then Eq. (44) also holds.

In Sec. IV B 2, we discuss the circumstances under which the limit in Eq. (44) applies, and can be achieved. The most obvious example, in which this limit can be achievable, is the case of an absorptive, background-free photon counter. In the presence of noise sources (such as thermal noise or detector noise), it may not be possible to attain this limit. Conversely, if a detection setup does not satisfy Eq. (45), then the FQL still places limits on its sensitivity, but these will depend on how much the fluctuations exceed the PQL value.

\section{B. SQL op-amp}

If we read out our signal using a phase-invariant, SQLlimited amplifier coupled weakly to the target (i.e., in opamp mode [35]), then from Appendix A 1, the SNR from an axion signal satisfies

$$
\mathrm{SNR}^{2} \leq\left(g B_{0} V_{b}\right)^{4} t \int_{0}^{\infty} \frac{d \omega}{2 \pi}\left(\frac{S_{\dot{a} \dot{a}}|\tilde{\chi}|^{2}}{|\tilde{\chi}|+\tilde{\chi}_{i}+S_{n}}\right)^{2}
$$

where $S_{n}$ summarizes the effects of any additional noise (beyond amplifier backaction, imprecision, and zero-point fluctuations) referred back to $A_{b}$. For example, if the target is subject to thermal noise at temperature $T$, we have $S_{n} \geq 2 n_{T} \tilde{\chi}_{i}$, where $n_{T}(\omega) \equiv\left(e^{\omega / T}-1\right)^{-1}$. We assume that the spatial profile of $J^{(a)}$ can be treated as constant in time, to begin with.

\footnotetext{
${ }^{7}$ While the thermal fluctuations of $A_{b}$ are set by $2 n_{T} \tilde{\chi}_{i}$, if the amplifier is coupled to other degrees of freedom, then the total effect of thermal noise on the output may be greater.
} 
If we are interested in a mass range $\Delta m \gg \delta \omega_{a}$ (i.e., a fractional mass range $\gg 10^{-6}$ ), then the quantity determining the axion-mass-averaged SNR squared is

$$
S \equiv \int_{\Delta m} d \omega|\tilde{\chi}|^{2}\left(\frac{1}{1+\left(1+2 n_{T}\right) \tilde{\chi}_{i} /|\tilde{\chi}|}\right)^{2} .
$$

The simplest form of response function is a single-pole oscillator, which has $\tilde{\chi}(\omega)=\frac{-1 / M}{\omega^{2}-\omega_{0}^{2}+i \omega \gamma}$, where $\omega_{0}$ is the undamped frequency, and $\gamma$ is the damping rate. Evaluating $S$ for this $\tilde{\chi}$, we find that if $\Delta m \geq\left(1+2 n_{T}\right) \gamma$, the integral is dominated by a bandwidth $\sim\left(1+2 n_{T}\right) \gamma$, and we have

$$
S \leq \frac{1}{2 V_{b}^{2} m^{2} \gamma f\left(n_{T}\right)}
$$

where

$$
f\left(n_{T}\right) \simeq \begin{cases}\frac{3}{4} & n_{T} \ll 1 \\ n_{T} & n_{T} \gg 1\end{cases}
$$

assuming that $\gamma \ll m$.

The dependence of Eq. (48) on $\gamma$ illustrates that the limit on $S$ will depend on what we assume about the damping properties of our system. As a simple example, suppose that an $A_{b}$ oscillation of amplitude $C$, for which the electric field energy is $U_{E}=\frac{1}{2} M \omega^{2}|C|^{2}$, results in a dissipated power of $P_{\text {diss }} \geq \gamma U_{E}$, for some $\gamma$ (for example, due to resistivity in the walls of a resonant cavity). The $A_{b}$ response to a monochromatic axion oscillation has $|C|^{2}=|\tilde{\chi}|^{2}|j|^{2}$, and the cycle-averaged absorbed power from the axion effective current is $P_{\text {abs }}=\frac{1}{2}|j|^{2} \omega \tilde{\chi}_{i}$. If the target is a passive system, then $P_{\text {abs }} \geq P_{\text {diss }}$, so we must have $\tilde{\chi}_{i} \geq M \omega \gamma|\tilde{\chi}|^{2}$. As we would expect, a single-pole response function with damping rate $\gamma$ has $\tilde{\chi}_{i}=M \omega \gamma|\tilde{\chi}|^{2}$.

We can immediately use the $\tilde{\chi}_{i} \geq M \omega \gamma|\tilde{\chi}|^{2}$ condition to place a limit on $S$, since

$$
S \leq \int_{\Delta m} d \omega|\tilde{\chi}|^{2} \leq \int_{\Delta m} d \omega \frac{\chi_{i}}{M \omega \gamma} \lesssim \frac{\pi}{2 V_{b}^{2} m^{2} \gamma} .
$$

This limit is only $\mathcal{O}(1)$ larger than the $n_{T}=0$ single-pole result [Eq. (48)]. In fact, we conjecture that a single-pole response function maximizes $S$, subject to the $\tilde{\chi}_{i} \geq M \omega \gamma|\tilde{\chi}|^{2}$ condition. With more restrictive conditions on $\tilde{\chi}$, we can prove this. For example, if $\tilde{\chi}$ can be written as a sum (with positive coefficients) of single-pole response functions with damping rate $\geq \gamma$, then since the integrand of $S$ is convex in the $\left(\tilde{\chi}_{r}, \tilde{\chi}_{i}\right)$ plane, $S$ is less than or equal to the sum of the integrals for the single-pole response functions. This form of response function is applicable when e.g., the dissipation rate for all of the relevant modes of the target is $\gamma$ (as would arise if we coupled together oscillators with damping rate $\gamma$ ).

To relate $S$ to the SNR for an axion DM signal, we can for simplicity take the axion signal to have a top-hat spectral form, $S_{\dot{a} \dot{a}} \simeq \frac{\pi \rho_{a}}{\delta \omega_{a}} \mathbf{1}_{\delta \omega_{a}}=\frac{\pi \rho_{a} Q_{a}}{m} \mathbf{1}_{\delta \omega_{a}}$. In this case,

$$
\begin{gathered}
\mathrm{SNR}^{2} \lesssim\left(g^{2} B_{0}^{2} V_{b}^{2} \rho_{a}\right)^{2} \frac{t \pi Q_{a}^{2}}{2 m^{2}} \times \\
\int_{\delta \omega_{a}} d \omega|\tilde{\chi}|^{2}\left(\frac{1}{1+\left(1+2 n_{T}\right) \tilde{\chi}_{i} /|\tilde{\chi}|}\right)^{2}
\end{gathered}
$$

So, averaging this over the $\Delta m$ axion mass range,

$$
\overline{\mathrm{SNR}^{2}} \lesssim \frac{\pi}{2}\left(g^{2} B_{0}^{2} V_{b} \rho_{a}\right)^{2} \frac{t Q_{a}}{m^{3} \Delta m \gamma}\left(\frac{S}{\left(V_{b}^{2} m^{2} \gamma\right)^{-1}}\right) .
$$

Using Eq. (50), this gives

$$
\overline{\mathrm{SNR}^{2}} \lesssim \frac{\pi^{2}}{4}\left(g^{2} B_{0}^{2} V_{b} \rho_{a}\right)^{2} \frac{t Q_{a}}{m^{3} \Delta m \gamma}
$$

If $S$ is maximized by a single-pole response, then

$$
\overline{\mathrm{SNR}^{2}} \lesssim \frac{\pi}{4}\left(g^{2} B_{0}^{2} V_{b} \rho_{a}\right)^{2} \frac{t Q_{a}}{m^{3} \Delta m \gamma f\left(n_{T}\right)} .
$$

Writing this in terms of the $\bar{P}$ expression from above,

$$
\overline{\mathrm{SNR}^{2}} \lesssim \frac{\bar{P}_{\max }^{2} Q_{a} t \Delta m}{\pi m^{3} \gamma f\left(n_{T}\right)}=\frac{\bar{P}_{\max }^{2} Q_{a} Q t \Delta m}{\pi m^{4} f\left(n_{T}\right)}
$$

where $Q \equiv \gamma / m$, and $\bar{P}_{\max }$ is the quantity from Eq. (41). As mentioned above, for each resonant configuration, the bandwidth contributing most of the $\mathrm{SNR}^{2}$ is $\sim\left(1+2 n_{T}\right) \gamma$, so we need to scan over multiple different configurations to have sensitivity over a wide mass range. Conversely, if $n_{T}$ is large enough that $\Delta m \lesssim 2 n_{T} \gamma$, then a single resonant configuration can cover the entire mass range approximately equally, giving

$$
\mathrm{SNR}^{2} \lesssim \frac{\pi}{8}\left(g^{2} B_{0}^{2} V_{b} \rho_{a}\right)^{2} \frac{t Q_{a}}{m^{3} \gamma^{2} n_{T}^{2}} .
$$

It should be noted that, to achieve the SNR bound in Eq. (46), which assumes minimum added noise $\bar{S}_{A_{b} A_{B}}^{\text {add }}=|\tilde{\chi}|$ (see Appendix A), it is necessary for the backaction spectral density to be $\bar{S}_{F F}=1 /(2|\tilde{\chi}|)$ at all $\omega$. If we had an amplifier connected directly to e.g., a high-quality-factor cavity, this would require its noise impedance to vary rapidly as a function of frequency. However, if we take $\bar{S}_{F F} \simeq$ const, then by choosing this constant value appropriately, we can do $\mathcal{O}(1)$ as well. For example, with a narrow-bandwidth single-pole response function, the optimum constant value of $\bar{S}_{F F}$ for $n_{T}=0$ is $\simeq M \omega \gamma$ (which is twice the optimum value on resonance), giving $S$ a factor $2 \pi / 9 \simeq 0.7$ smaller than choosing the optimum $\bar{S}_{F F}$ at every frequency.

Another point to note is that it may be possible to place tighter bounds on $S$ by analyzing the system's dissipation more carefully. In particular, if the system's response to an axion forcing has stored energy $U>U_{E}$, and $P_{\text {diss }} \geq \gamma U$, then $P_{\text {abs }} \geq P_{\text {diss }}$ implies that 


$$
\tilde{\chi}_{i} \geq \frac{U}{U_{E}} M \omega \gamma|\tilde{\chi}|^{2}
$$

and in effect, one can replace $\gamma$ by $\gamma U / U_{E}$ in the expressions above. We discuss an example of this in Sec. IV C, where we consider oscillations in the quasistatic regime, for which the stored magnetic field energy is much larger than the energy in the electric fields.

The above expressions are only valid when the relevant integration times are long compared to the inverse bandwidth of spectral features. Looking at the $n_{T} \ll 1$ limit, if we consider a single-pole resonator with $Q \gg Q_{a}$, then we need at least $\Delta m / \delta \omega_{a}$ tuned configurations to have sensitivity across the $\Delta m$ mass range, so we can spend at most $t_{1}=t_{\text {tot }} \frac{\delta \omega_{a}}{\Delta m}$ in each of them. If $t_{1} \lesssim Q / \nu$, then we do not resolve the resonator bandwidth, and the formulas above do not hold. In particular, if we fix $t_{\text {tot }}$, then for $Q \gtrsim \nu t_{1}$, we do not expect the SNR to continue improving with increasing $Q$ (this limiting $Q$ is often large, but may sometimes be of practical relevance [14]). Plugging $Q \simeq$ $\nu t_{1}$ into Eq. (56), which should be parametrically (though not numerically) valid, we obtain

$$
\overline{\mathrm{SNR}^{2}} \lesssim \frac{1}{3 \pi^{2}}\left(\frac{\bar{P}_{\text {max }} t_{\text {tot }}}{m}\right)^{2} \simeq\left(0.2 \bar{N}_{\max }\right)^{2}
$$

so the SNR limit is parametrically the same as the PQL. In simple cases, such as when the backaction power spectral density is frequency independent, this corresponds to the quantum fluctuations of $A_{b}$ satisfying the PQL sum rule [at least to $\mathcal{O}(1)]^{8}$

If the spatial profile of $J^{(a)}$ is not constant, then as discussed in Sec. II D, we can consider the time variation in an orthogonal set of spatial profiles. This is necessary if the axion coherence length is smaller than the scale of the experiment (or the extent of the background magnetic field, whichever is smaller), i.e., if $\nu_{a} \gtrsim\left(v_{a} L\right)^{-1} \sim$ $300 \mathrm{GHz}(\mathrm{m} / L) .{ }^{9}$ The SNRs from these orthogonal spatial profiles will add in quadrature.

\footnotetext{
${ }^{8}$ In the framework of [35], the fluctuations of $A_{b}$ can be made large by increasing the amplifier-target coupling. However, this also increases the amount we learn about $A_{b}$ from the amplifier output; to determine the quantum fluctuations of $A_{b}$, we need to condition on the observed output of the amplifier (cf. the analysis of backaction evasion in [54]).

${ }^{9}$ In contrast to how the fluctuation spectrum can be concentrated into a narrow-bandwidth peak, at the expense of surrounding frequencies, the frequency-averaged fluctuations for each orthogonal spatial mode are fixed by its spatial profile, and cannot be concentrated into one spatial mode at the expense of others. In the impulse picture, if we imagine a set of simultaneous impulses at different spatial points, then causality prevents the energy absorbed from depending on their relative signs etc., whereas the responses to impulses at different times (picking out a specific frequency) can interfere with each other.
}

\section{Isolated amplifier}

For the op-amp coupling considered in the previous section, the amplifier's backaction noise couples to $A_{b}$. Another way to couple an amplifier to the target is to isolate it, so that its backaction noise has no effect on $A_{b}$. This is common at microwave frequencies, where an amplifier is usually isolated behind a circulator, with its backaction absorbed by a cold load $[35,38,55,56]$. The effect on the target is as if we simply connected it to the cold load.

In contrast to the op-amp case, where the coupling between the amplifier and the target is taken to be very weak, achieving a good SNR in isolated configurations requires stronger couplings to the target, which significantly affect its damping properties [35]. To find a SNR limit, we can adopt a formal perspective in which we view all of the degrees of freedom apart from $A_{b}$ as making up the "amplifier," and write $H_{\text {int }}=A_{b} \hat{F}$. Then, if the target is in its ground state (i.e., its temperature and that of the cold load are negligible), the fluctuation spectrum of $\hat{F}$ must be such that $S_{A_{b} A_{b}}=2 \tilde{\chi}_{i} \Theta(\omega)$, so $S_{F F}=2 \frac{\tilde{\chi}_{i}}{\mid \tilde{\chi}^{2}} \Theta(\omega)$. Using the analysis from [35], this implies that the total noise at the amplifier output is $\bar{S}_{A_{b} A_{b}}^{\text {tot }} \geq \frac{|\tilde{\chi}|^{2}}{2 \tilde{\chi}_{i}} \cdot{ }^{10}$ Consequently,

$$
\mathrm{SNR}^{2} \leq\left(g B_{0} V_{b}\right)^{4} t \int_{0}^{\infty} \frac{d \omega}{2 \pi}\left(2 S_{\dot{a} \dot{a} \tilde{\chi}_{i}}\right)^{2}
$$

in agreement with the limits of the circulator-plus-cold-load analyses from $[14,25]$. If we assume $\tilde{\chi}_{i} \geq M \omega \gamma|\tilde{\chi}|^{2}$, as discussed in the previous subsection, then $\tilde{\chi}_{i}^{2} \leq|\chi|^{2} \leq$ $\tilde{\chi}_{i} /(M \omega \gamma)$, so we have

$$
\int_{\Delta m} d \omega \tilde{\chi}_{i}^{2} \leq \frac{\pi}{2 M^{2} \omega^{2} \gamma}
$$

Consequently, the axion-mass-averaged $\mathrm{SNR}^{2}$ is bounded by

$$
\overline{\mathrm{SNR}^{2}} \lesssim \pi^{2}\left(g^{2} B_{0}^{2} V_{b} \rho_{a}\right)^{2} \frac{t Q_{a}}{m^{3} \Delta m \gamma} .
$$

This has the same parametric form as Eq. (54). For a singlepole response function, we have

$$
\overline{\mathrm{SNR}^{2}} \lesssim \frac{\pi}{4}\left(g^{2} B_{0}^{2} V_{b} \rho_{a}\right)^{2} \frac{t Q_{a}}{m^{3} \Delta m \gamma}
$$

which is $\mathcal{O}(1)$ less than Eq. (62). As in the previous subsection, a single-pole response is optimal if the response function is a convex combination of single-pole response

\footnotetext{
${ }^{10}$ For an ideal quantum amplifier, $\bar{S}_{A_{b} A_{b}}^{I} \bar{S}_{F F} \geq 1 / 4$. However, in our case $S_{F F}=0$ for $\omega<0$, whereas $S_{I I}$ is even, which implies that there must be wasted information in the FI correlation [35]. In particular, by feeding back some of the output, the backaction could be made symmetric, decreasing $\bar{S}_{F F}$ by a factor $1 / 2$. Consequently, in our case, $\bar{S}_{A_{b} A_{b}}^{I} \bar{S}_{F F} \geq 1 / 2$. Thus, $\bar{S}_{A_{b} A_{b}}^{I} \geq \frac{1}{2 \bar{S}_{F F}}=\frac{|\tilde{\chi}|^{2}}{2 \tilde{\chi}_{i}}$.
} 
functions with damping rate $\geq \gamma$, and we conjecture that it is also optimal in the more general case of $\tilde{\chi}_{i} \geq M \omega \gamma|\tilde{\chi}|^{2}$.

If the integration time becomes long compared to the inverse bandwidth of spectral features, $Q \sim \nu t_{1}$, then we again obtain $\mathrm{SNR} \sim \bar{N}_{\max }$. An isolated amplifier satisfies the PQL assumptions, so this bound necessarily holds.

It should be emphasized that the $\gamma$ in Eqs. (62) and (63) refers to the damping rate with the readout system connected to the target, and not just to the intrinsic damping $\gamma_{\text {int }}$ in the target itself (whereas $\gamma=\gamma_{\text {int }}$ in the op-amp case). Analyzing the case of a circulator plus a cold load, as in $[14,25]$, shows that for a given intrinsic dissipation rate $\gamma_{\text {int }}$ associated with the target, the axion-mass-averaged SNR squared is optimized for $\gamma_{\text {det }}=2 \gamma_{\text {int }}$, where $\gamma=$ $\gamma_{\text {det }}+\gamma_{\text {int }}$ (at zero temperature, and for a single-pole response function). This leads to a SNR limit $\mathcal{O}(1)$ worse than Eq. (62).

So far, we have assumed that the target is in the ground state. If the target and the cold load have the same nonzero temperature, then an analogous analysis can easily be performed, incorporating thermal fluctuations for $A_{b}$. However, if the cold load is maintained at a different temperature from the target's environment, the situation is slightly more complicated. As analyzed in [14,24,25], if the cold load is at a lower temperature than the target's environment, and the dissipation in the target system is fixed, it is beneficial to "overcouple" to the amplifier, increasing the damping rate of the target excitation. If all temperatures were negligibly small, this would result in worse sensitivity, but because it also reduces the thermal noise reaching the detector, its overall effect is to improve the SNR. From $[14,24,25]$, for $n_{T} \gg 1$, where $T$ is the temperature of the target, the resulting $\mathrm{SNR}^{2}$ is suppressed by $\sim 1 / n_{T}$ compared to the $T=0$ value.

It should be possible to give a more detailed analysis of isolated amplifier setups than attempted in this section. Our main aim here was to show that the SNR obtained from a single-pole resonator is $\mathcal{O}(1)$ optimal (at least at zero temperature).

\section{Quantum-limited op-amp}

As discussed in Appendix A 1, if it is possible to optimize the correlations between a linear amplifier's backaction and imprecision noise, then the minimum added noise referred back to the measured variable $x$ is $S_{x x}^{\text {add }}(\omega) \geq$ $\left|\tilde{\chi}_{i}(\omega)\right|$ (as opposed to $S_{x x}^{\text {add }} \geq|\tilde{\chi}|$ for uncorrelated noise). Consequently, for an amplifier connected in op-amp mode,

$$
\mathrm{SNR}^{2} \leq\left(g B_{0} V_{b}\right)^{4} t \int_{0}^{\infty} \frac{d \omega}{2 \pi}\left(\frac{S_{\dot{a} \dot{a}}|\tilde{\chi}|^{2}}{2 \tilde{\chi}_{i}+S_{n}}\right)^{2} .
$$

Assuming thermal noise, $S_{n}=2 n_{T} \tilde{\chi}_{i}$, and taking $\tilde{\chi}_{i} \geq M \omega \gamma|\tilde{\chi}|^{2}$, we have

$$
\mathrm{SNR}^{2} \leq \frac{1}{8 \pi}\left(g^{2} B_{0}^{2} V_{b}\right)^{2} t \int_{0}^{\infty} d \omega\left(\frac{S_{\dot{a} \dot{a}}}{\omega \gamma\left(1+n_{T}\right)}\right)^{2} .
$$

If we take $S_{\dot{a} \dot{a}}$ to have top-hat form, as above, then

$$
\mathrm{SNR}^{2} \lesssim \frac{\pi}{8}\left(g^{2} B_{0}^{2} V_{b} \rho_{a}\right)^{2} t \frac{Q_{a}}{m_{a}^{3} \gamma^{2}\left(1+n_{T}\right)^{2}}
$$

This has the same form as the SQL expression in Eq. (57), when $\Delta m \lesssim\left(1+2 n_{T}\right) \gamma$; this is as expected, since in both cases, the added noise is dominated by the thermal plus vacuum noise. If $\Delta m \gtrsim\left(1+2 n_{T}\right) \gamma$, then we can improve over the SQL limit by $\sim \frac{\Delta m}{2 n_{T} \gamma}=\frac{Q}{2 n_{T}} \frac{\Delta m}{m}$ (for $n_{T} \gg 1$ ). This is simply the ratio between the sensitivity bandwidth for the SQL-limited amplifier, $\sim\left(1+2 n_{T}\right) \gamma$, and the broadband $(\sim \Delta m)$ sensitivity for the QL-limited case. On resonance, where $\tilde{\chi}$ is purely imaginary, we achieve the QL limit by having uncorrelated backaction and imprecision noise, so we expect the QL and SQL limits to coincide in this case.

As the above limits show, the sensitivity limit for a quantum-limited amplifier is set by how well we can isolate the target system from the dissipative environment, and so reduce $\gamma$. There does not have to be a sensitivity/bandwidth trade-off, as occurs in the SQL and PQL cases, since as $\tilde{\chi}_{i}$ decreases, the ZPF noise also decreases; a setup that saturates the QL at all frequencies is naturally broadband. Near a narrow resonance, this would require the backaction/imprecision correlations to change very fast as a function of frequency, but far from the resonance, the required change is slower.

Conceptually, there are various amplification schemes that can make use of correlated backaction and imprecision noise to attain the QL over some frequency range, e.g., the driven nonlinear cavity method proposed in [57]. At higher frequencies $(\gtrsim \mathrm{GHz})$, this is usually practically difficult (in particular, it is not compatible with isolation mechanisms such as circulators, as discussed above). However, at lower frequencies, SQUID amplifiers can attain near-QL performance, in some circumstances [42]. We discuss axion detection at low frequencies $(\ll \mathrm{GHz})$ in Sec. IV C.

The fact that a QL amplifier can have better sensitivity than the PQL limit, in some regimes, corresponds to the amplifier enhancing the frequency-averaged quantum fluctuations of $A_{b}$ (similar to how backaction evasion effectively drives an oscillator into a squeezed state [54]). For a single-pole response function, the $A_{b}$ quantum fluctuations have the usual value on resonance, but are unsuppressed off resonance, corresponding to the broadband sensitivity described above.

\section{E. Up-conversion}

We can generalize the power absorption calculations above to a time-dependent magnetic field. In this case, 


$$
\begin{gathered}
P_{m} \simeq \frac{g^{2} V_{b}^{2}}{(2 \pi)^{2}} \int_{-\infty}^{\infty} d \omega \omega \tilde{\chi}_{i}(\omega)\left(S_{\dot{a} \dot{a}} * S_{B B}\right)_{\omega} \\
=\frac{g^{2} V_{b}^{2}}{(2 \pi)^{2}} \int_{-\infty}^{\infty} d \omega S_{\dot{a} \dot{a}}(\omega)\left(\left(\omega \tilde{\chi}_{i}\right) * S_{B B}\right)_{\omega}
\end{gathered}
$$

where $S_{B B}$ is the spectral density of $B_{0}(t)$. Using Eq. (38),

$$
\begin{aligned}
\int d m P_{m} & \simeq \frac{g^{2} V_{b}^{2} \rho_{a}}{4 \pi} \int_{-\infty}^{\infty} d \omega\left(\left(\omega \tilde{\chi}_{i}\right) * S_{B B}\right)_{\omega} \\
& \simeq g^{2} V_{b} \rho_{a} \overline{B_{0}^{2}} \frac{\pi}{2}
\end{aligned}
$$

where $\int_{-\infty}^{\infty} d \omega S_{B B}(\omega)=2 \pi \overline{B_{0}^{2}}$, so $\sqrt{\overline{B_{0}^{2}}}$ is the rms $B_{0}$ value. In the case of a static $B_{0}$ field, this reproduces Eq. (40).

There are a number of qualitatively distinct cases, depending on the oscillation frequencies of the magnetic field and the axion signal. For a $B_{0}$ oscillation at frequency $\omega_{B}$, an axion oscillation at $\omega_{a}$ will give a forcing at sum and difference frequencies, $\omega_{B}+\omega_{a}$ and $\left|\omega_{B}-\omega_{a}\right|$. To start with, we will consider the up-conversion case where $\omega_{B} \gg \omega_{a}$, so that both sum and difference frequencies are $\gg \omega_{a}$.

The power absorbed from single-frequency axion and $B_{0}$ oscillations is set by

$P \simeq \frac{1}{4} g^{2} V_{b}^{2} B_{0}^{2} \rho_{a} \omega_{B}\left(\tilde{\chi}_{i}\left(\omega_{B}+m_{a}\right)+\tilde{\chi}_{i}\left(\omega_{B}-m_{a}\right)\right)$

(this is valid over times $\gg m_{a}^{-1}$, so that we resolve the two different frequencies). Averaging this over an axion mass range $\Delta m$, we obtain

$$
\bar{P} \lesssim \frac{g^{2} \rho_{a} V_{b} B_{0}^{2}}{\Delta m} \frac{\pi}{8}=\frac{g^{2} \rho_{a} V_{b} \overline{B_{0}^{2}}}{\Delta m} \frac{\pi}{4}
$$

which can be saturated by concentrating $\chi_{i}$ into an $\sim \Delta m$ range either above or below $\omega_{B}$ (or in both ranges). This only represents half of the total mass-integrated power absorbed. Since axions at $m_{a} \simeq 2 \omega_{B}$ will also excite a target mode at $\simeq \omega_{B}$, the other half of the absorption is at these, much higher, axion masses.

The PQL detectability is again set by the expected number of quanta absorbed, which is $N_{a} \simeq P t / \omega_{B}$. Compared to a static-field experiment with the same $P$, an up-conversion experiment absorbs fewer but higherenergy quanta. ${ }^{11}$ The SQL limit for a single-pole response

\footnotetext{
${ }^{11}$ In the above, we have considered the power absorbed from the axion-induced effective current, since this is the quantity that is usually relevant for detection. For a static background magnetic field, this is the same as the power absorbed from the axion DM field. However, for an up-conversion experiment, each axion quantum absorbed results in a photon at frequency $\sim \omega_{B} \gg \omega_{a}$; the extra energy comes from the oscillating background magnetic field.
}

(which is parametrically the same as the isolated-amplifier limit) is

$\overline{\mathrm{SNR}^{2}} \leq \frac{1}{\pi} \bar{P}_{\max }^{2} \frac{\Delta m}{m} \frac{t Q_{a} Q_{1}}{\omega_{1}^{3} f\left(n_{T}\left(\omega_{1}\right)\right)} \simeq \frac{1}{\pi} \bar{P}_{\max }^{2} \frac{\Delta m}{m} \frac{t Q_{a} Q_{1}}{T \omega_{1}^{2}}$

where $Q_{1} \equiv \omega_{1} / \gamma$, and the second equality is for $T \gg \omega_{1}$. For $n_{T} \ll 1$, taking $Q$ as large as it can be while still resolving the resonator bandwidth gives $\overline{\mathrm{SNR}^{2}} \sim$ $\left(\frac{\bar{P}_{t_{\text {tot }}}}{\omega_{1}}\right)^{2} \sim \bar{N}^{2}$, in analogy to Eq. (59). A QL-limited amplifier could improve on the SQL by the usual $\sim \frac{\Delta m}{2 n_{T} \gamma}$ factor. However, as we will discuss in Sec. IV D, up-conversion experiments are most interesting at $\omega_{1} \gtrsim \mathrm{GHz}$, and utilizing backaction/imprecision correlations is difficult there.

As the limits above indicate, up-conversion experiments have reduced sensitivity, compared to ideal staticfield experiments with the same $\bar{U}_{B}$. However, as we will discuss in Sec. IV C, for low enough axion masses, it is hard for static-field experiments to attain the power and sensitivity bounds from above, due to being in the quasistatic regime. Consequently, up-conversion experiments can have a parametric advantage in absorbed power, and sensitivity, for low axion masses.

\section{F. Down-conversion}

We can also consider other frequency combinations. If $\omega_{B} \ll m_{a}$, then the situation is basically the same as the static-field case. The remaining distinct case is when $\omega_{B} \simeq m_{a}$, so that the difference frequency is small, $\omega_{s}=\left|\omega_{B}-m_{a}\right| \ll m_{a}$.

In this case, which we label "down-conversion," it is possible to attain the $\bar{P}$ bound from Eq. (70), by concentrating $\tilde{\chi}_{i}$ at low frequencies $\sim \omega_{s}$. Then, $\left(\omega \tilde{\chi}_{i}\right) * S_{B B}$ is entirely at frequencies $\sim \omega_{B}$. Thus, we obtain the same converted power limit as for a static-field experiment.

However, since $N_{a}=P t / \omega_{s}$, by taking $\omega_{s}$ smaller and smaller, we can make the PQL sensitivity better and better (for integration times long enough to resolve $\left.\omega_{s}\right)$. Physically, this is the converse of the up-conversion case; we maintain the same converted power, but this corresponds to absorbing more low-energy quanta. ${ }^{12}$ As per Eq. (73), the SQL-limited SNR is $\propto 1 / \omega_{s}$, for given $U_{B}, Q$, and $T \gtrsim \omega_{s}$ (and $\propto \omega_{s}^{-3 / 2}$ for $T \lesssim \omega_{s}$ ).

Thus, at least in principle, we can improve our sensitivity by making $\omega_{s} \ll m_{a}$. However, as we discuss in Sec. IV E, there are usually serious practical obstacles to obtaining an advantage in this way.

\footnotetext{
${ }^{12}$ From the point of view of the axion field, the $A \cdot B$ term it is interacting with oscillates at a frequency $\sim m_{a}$, whereas the effective current $g \dot{a} B$ oscillates at $\sim \omega_{s}$; the energy change of the axion field is therefore greater than the energy gain in the target mode, with the difference made up by the $B_{0}$ oscillation.
} 


\section{DM DETECTION EXPERIMENTS}

In the previous section, we analyzed limits on the power absorption and sensitivity of idealized axion DM detection experiments, in terms of the magnetic field maintained in the experiment. In this section, we will investigate the sensitivities of more specific experimental setups, and how they relate to these theoretical limits.

\section{A. Static background field: Power absorption}

From Eq. (41), a static-field experiment covering an axion mass range $\Delta m$ must have

$$
\min _{m \in \Delta m} P \leq \pi \frac{g_{a \gamma \gamma}^{2} \rho_{a} \bar{U}_{B}}{\Delta m}
$$

if it is in the linear response regime, and if $\tilde{\chi}_{i}(\omega) \geq 0$ for $\omega>0 .{ }^{13}$ For experiments with $m_{a} \gtrsim L^{-1}$, where $L$ is the length scale of the shielded volume, it is easy to see that the usual EM field modes $\mathcal{O}(1)$-saturate the sum rules, for slowly varying $B_{0}$ profiles. Consequently, the signal power for cavity and dielectric haloscopes is parametrically given by Eq. (74). For example, a cavity haloscope has an onresonance, fully-rung-up signal power of

$$
P_{\text {sig }}=C g^{2} B_{0}^{2} V Q \frac{\rho}{m}
$$

where $Q$ is the quality factor of the cavity mode, and

$$
C \equiv \frac{\left|\int d V E \cdot B_{0}\right|^{2}}{\int d V\left|B_{0}\right|^{2} \int d V|E|^{2}}
$$

is the normalized overlap between the background magnetic field and the electric field of the mode. Geometrically, $C \leq 1$. If $Q \gg 1$, then the converted power is approximately a Lorentzian function of the axion mass, so if we want to cover a small mass range $\Delta m$ with cavity configurations tuned to different resonant frequencies, we have

$$
\bar{P} \simeq \frac{g^{2} B_{0}^{2} V_{b} \rho}{\Delta m} \frac{\pi}{2} C
$$

as expected from Eq. (74). ADMX [16,17] uses the $\mathrm{TM}_{010}$ mode of a cylindrical cavity, which has $C \simeq 0.68$ (ignoring perturbations from the tuning rods), so is $\mathcal{O}(1)$ optimal for its cavity volume. The rest of the sum-rule-determined absorption is into other cavity modes; these will generally be at different frequencies, so will not be useful for searching a small axion mass range (though they may be used to perform simultaneous searches in different mass

\footnotetext{
${ }^{13}$ More generally, Eq. (74) applies if $\tilde{\chi}_{i}$ is well approximated, in the frequency range $\Delta m$, by the response function $\tilde{\chi}_{i}{ }^{\prime}$ for a system which has $\tilde{\chi}_{i}{ }^{\prime} \geq 0$ for $\omega \geq 0$.
}

ranges, e.g., ADMX's usage of the $\mathrm{TM}_{020}$ mode alongside $\mathrm{TM}_{010}$ [55]).

For a dielectric haloscope with alternating half-wave layers of refractive indices $n_{1}, n_{2}$, the average signal power over an axion mass range $\Delta m$ around the half-wave frequency is [21]

$$
\bar{P}=\frac{4}{\pi} \frac{g^{2} B_{0}^{2} V \rho}{\Delta m}\left(\frac{1}{n_{2}}-\frac{1}{n_{1}}\right)^{2} .
$$

If we take $n_{1}=1, n_{2} \nearrow \infty$, then this is $\sim 8 / \pi^{2}=0.81$ of the sum-rule limit. The rest of the response function will be at other axion masses. For a half-wave stack, this will mostly be at odd multiples of the half-wave frequency [21]. Since the absorbed power at each multiple drops off $\propto 1 / \omega^{2}$, and $1 /\left(\sum_{n=0}^{\infty} \frac{1}{(2 n+1)^{2}}\right)=\frac{8}{\pi^{2}}$, this agrees with the $8 / \pi^{2}$ fraction absorbed into the first multiple that we found from Eq. (78).

At microwave frequencies, it is relatively simple to find low-loss transparent dielectrics, which can almost saturate these bounds; for example, the MADMAX [20,58] proposal currently aims to use $\mathrm{LaAlO}_{3}$ layers, with permittivity $\epsilon \simeq 24$. However, at higher frequencies, it may be difficult to find suitable dielectrics with large $n$. For example, in the energy range $\sim 0.2-0.5 \mathrm{eV}$, a potentially practical pair of transparent dielectrics is $\mathrm{Si}(n \simeq 3.4)$ and $\mathrm{Al}_{2} \mathrm{O}_{3}(n \simeq 1.77)$. This gives a suppression of $\frac{8}{\pi^{2}}\left(\frac{1}{n_{2}}-\frac{1}{n_{1}}\right)^{2} \simeq 0.07$ for the absorbed power, relative to the volume-limited value.

In addition, for a small number of layers, the shielded volume required may be significantly larger than the dielectric-occupied volume, e.g., to allow space for the focusing optics. In that sense, the experiment is even further from optimality. ${ }^{14}$ However, such experiments can still be efficient compared to other high-frequency proposals, such as dish antennas [60]. For example, if we consider the $\mathrm{Si} / \mathrm{SiO}_{2}$ chirped stacks with reach shown in Fig. 2 [each of these has 100 periods, covers an octave in axion mass range, and has area $\sim(18 \mathrm{~cm})^{2}$ ], an equivalent reach could be obtained from an $\sim \mathrm{m}^{2}$ dish antenna in the same background magnetic field. This dish would require a significantly larger volume for the magnetic field, and could only concentrate the emitted photons onto a significantly larger area, complicating detection. If larger volumes of photonic material could be fabricated, or larger refractive index contrasts achieved, the comparison would be even more favorable to the layered materials.

The cavity and dielectric haloscope examples above achieved $\bar{P}$ values at $\mathcal{O}(1)$ of the limit. An example of a target that could theoretically attain the limit almost fully (for a uniform background magnetic field, within a narrow axion mass range) is a uniform plasma, of large extent

\footnotetext{
${ }^{14}$ Techniques such as using microlens arrays rather than a single lens [59] may help with volume utilization.
} 
compared to the axion Compton wavelength (as analyzed in [61]). It should be noted that the wire metamaterials proposed in [61] most likely do not have this property, and have power absorption at $\mathcal{O}(1)$ of the limit, similar to a cavity haloscope.

The sum-rule limits explain some basic properties of axion conversion rates in experiments, in particular, the power/bandwidth trade-off, and the similar axion-massaveraged signal powers attained from different types of experiments with comparable volumes and magnetic fields. They also imply less obvious facts. For example, in dielectric haloscopes, one can enhance the conversion rate near a particular frequency by making the dielectric permittivity $\epsilon$ of some layers $\epsilon<1$, e.g., by operating near an optical resonance (the stack configuration of the molecular absorption proposal in [62] is an example of this). However, the sum rules tell us that this is compensated for by lower powers at other frequencies, and cannot improve the average power over an $\mathcal{O}(1)$ axion mass range.

As mentioned in Sec. I A, static-field experiments for $m_{a} \ll L^{-1}$ generally suffer a quasistatic suppression, and the maximum power absorbed is suppressed by $\sim\left(m_{a} L\right)^{2}$. We defer discussion of this regime to Sec. IV C.

\section{B. Detectability}

\section{Linear amplifiers}

Section III showed that, for systems satisfying the PQL assumptions, or for readout via a $\mathrm{SQL}$ or isolated amplifier, the theoretical detectability limits are closely related to the limit on $\bar{P}$. With SQL op-amp readout, it is easy to imagine idealized setups that attain the SNR bound from Eq. (55), to $\mathcal{O}(1)$. For example, in a cavity haloscope experiment, we could connect an antenna to a SQL-limited current amplifier. In the case of a linear amplifier isolated behind a circulator and cold load, it is also easy, in principle, to attain the bounds from Sec. III C to $\mathcal{O}(1)$ [14].

There have been claims in the literature that simple feedback schemes can give enhanced sensitivities, for example, the digital feedback scheme of [63]. Since the feedback applied in [63] is entirely coherent state, and a SQL (or circulator-isolated) readout system seems to be assumed, the bounds derived above should apply. In particular, the thermal-noise-limited sensitivity cannot be improved by adding a known, coherent signal to the mode. Moreover, attempting to cover multiple frequency ranges in parallel results in worse theoretical sensitivity than tuning a narrow resonance over time, as per the results of Sec. III.

\section{2. $P Q L$}

In the PQL case, it is also simple to describe setups that can (in principle) attain the $N_{a}=\bar{P} t / m_{a}$ limit, for example, if we have no added noise ( $T \ll m_{a}$ etc.), and almost all of the converted power is absorbed by a background-free photon counter.

One motivation for discussing the PQL, as opposed to specializing to e.g., absorptive photon counters directly, is that it can also apply to experiments using different kinds of target excitations, such as phonons [64], electron-hole pairs [65], or more complicated quasiparticles [66] (and different detection schemes such as optical homodyne detection). While a detection setup always includes some components that are not in thermal equilibrium (e.g., amplifiers), in many cases, these are connected to the target system via a damping-type coupling. Examples include absorptive photodetectors, or sensors isolated behind a circulator with a matched load. In these cases, the quantum fluctuations of the target system are as if the sensor were replaced by an equivalent passive load. Consequently, the experiment's sensitivity should be bounded by the PQL (for example, this applies directly to the case of a SQL amplifier isolated behind a circulator, as discussed above).

As mentioned in Sec. I A 1, it is possible to violate the PQL by preparing the EM field in a "nonclassical" state, e.g., a Fock state or a squeezed state. It is also the case that some detection schemes push the target into a PQLviolating state; examples include backaction evasion [54], QND photon counting, and (as per the QL discussions above) correlated backaction/imprecision noise.

\section{Quasistatic regime}

When $m_{a} \ll L^{-1}$, it is significantly more difficult to attain the sum-rule bounds with a static-field experiment. This is because the EM fields are naturally in the quasistatic regime at frequencies $\ll L^{-1}$, and their $A$ fluctuations are suppressed. The magnitude of the linear response function is similarly suppressed, so the amplifier SNR is also affected.

We can demonstrate this suppression, somewhat heuristically, by considering the fluctuations of the energy in the electromagnetic field. The rate of change of EM field energy is

$$
P_{\mathrm{EM}}=-\int d V E \cdot J
$$

In Lorenz gauge, $\nabla \cdot A=-\partial_{t} A_{0}$, we have $\partial_{\mu} \partial^{\mu} A^{\nu}=$ $\left(\partial_{t}^{2}-\nabla^{2}\right) A^{\nu}=J^{\nu}$. If we are considering very low frequencies, $\omega \ll L$, then since the $A^{\nu}$ and $J^{\nu}$ fields are localized on scales $\sim L$, we have $\nabla^{2} A^{\nu} \simeq-J^{\nu}$. Hence,

$$
P_{\mathrm{EM}} \simeq \int d V E \cdot\left(\nabla^{2} A\right)
$$

We can ignore the low-frequency condition for a moment, and take the expectation value of Eq. (80)'s rhs, in the ground state. As per Sec. II D, the terms 
corresponding to spatially orthogonal $A$ profiles add independently, and have the same sign. Since

$$
-\int d V b \cdot\left(\nabla^{2} b\right)=\int \frac{d^{3} k}{(2 \pi)^{3}} k^{2}\left|b_{k}\right|^{2} \gtrsim L^{-2} V_{b}
$$

we have

$$
-i \int d V\left\langle E_{b} \cdot\left(\nabla^{2} A_{b}\right)\right\rangle \gtrsim-i\left\langle E_{b} A_{b}\right\rangle \frac{V_{b}}{L^{2}}=\frac{1}{2 L^{2}} .
$$

Similarly, if we consider the fluctuations within a particular frequency range, we have

$$
\Delta P_{\mathrm{EM}} \gtrsim \frac{V_{b}}{L^{2}} \frac{1}{2 \pi} \int_{\Delta \omega} d \omega \omega S_{A_{b} A_{b}}(\omega) .
$$

Hence, if the sum rule were $\mathcal{O}(1)$-saturated by the lowfrequency fluctuations, then the low-frequency fluctuations of $P_{\mathrm{EM}}$ would be $\gtrsim \frac{1}{2 L^{2}}$, in the ground state. In contrast, if the low-frequency EM modes behave like harmonic oscillators, then $P_{\mathrm{EM}} \sim \omega^{2}$. Hence, in the latter case, the average value of $\bar{S}_{A_{b} A_{b}}$ must be suppressed by $\sim(\omega L)^{2}$ from its sumrule-limited value.

We can gain some physical intuition for this suppression by splitting the quasistatic electric field into a "Coulomb" part $E_{C}=-\nabla A_{0}$, and a "Faraday" part $E_{F}=-\partial_{t} A$ (again, in Lorenz gauge). The Coulomb part can have large fluctuations (e.g., for oscillations involving a capacitor), but $E_{C}$ has zero integrated overlap with $B_{0}$. Conversely, $E_{F}$ is naturally $\sim(\omega L) B$, where $B$ is the magnetic field fluctuation. Hence, if the ground-state magnetic field fluctuations in a mode carry $\sim$ one quantum of energy, which is the case for harmonic-oscillator-like modes, then the $E_{F}$ fluctuations will be suppressed, as will the $A$ fluctuations.

Given this suppression, a static-field experiment searching for an axion over a small mass range $\Delta m$ around $m_{a}$ must have

$$
\min P \lesssim \frac{g^{2} B_{0}^{2} V_{b} \rho_{a}}{\Delta m}\left(m_{a} L\right)^{2}
$$

This scaling can be confirmed by calculations for specific experimental setups. In [67], the response of a small cavity in a constant magnetic field was computed, and in [23] a toroidal magnetic field was considered, both displaying the expected $\sim(m L)^{2}$ suppression.

The suppressed power absorption directly limits the sensitivity of PQL-limited searches. For SQL searches, the $\sim(m L)^{2}$ suppression of the frequency-averaged $\tilde{\chi}_{i}$ value contributes an $\sim(m L)^{2}$ suppression in $\mathrm{SNR}^{2}$, while another factor comes from $U_{E} / U \sim(m L)^{-2}$ in Eq. (58), since the electric field fluctuations are only an $\sim(m L)^{2}$ fraction of the total energy of an $\omega \simeq m$ oscillation. For QL searches, the $U_{E} / U$ suppression itself suppresses the $\mathrm{SNR}^{2}$ by $\sim(m L)^{4}$. Consequently, for all of these types of searches, the $g$ sensitivity is suppressed by $\sim(m L)^{-1}$ compared to its higher-frequency scaling. ${ }^{15}$

References [24,25] conducted a detailed analysis of SQL-limited detection in the quasistatic regime, assuming a given axion-induced flux coupled through a pickup loop of given inductance. In agreement with our analysis, the $\mathrm{SNR}^{2}$ limit they obtain is parametrically given by Eq. (55) suppressed by $(m L)^{4}$.

As well as the resonant approaches of $[24,25]$, broadband approaches to low-frequency axion DM detection have been proposed, such as ABRACADABRA [23]. This intends to use SQUID amplifiers, which can, in some circumstances, achieve near-QL sensitivity [42]. If a QL-limited broadband experiment could be realized, it would have superior sensitivity to a SQL-limited resonant search, as discussed in Sec. IIID (the comparison of resonant to broadband approaches in [24,25] was based on both being SQL limited). In practice, achieving the required amplifier properties would likely be very difficult (for a large pickup loop, with a correspondingly large inductance, an amplifier with a matching noise impedance would be required). Figure 1 compares the PQL, SQL, and QL sensitivities in the quasistatic regime, illustrating these differences.

\section{Evading quasistatic limits}

It should be noted that, to escape the "quasistatic" regime where $\nabla^{2} A \simeq-J$, not all of the dimensions need to be large. For example, the short cylinders proposed in [63] can attain the sum-rule bound to $\mathcal{O}(1)$, as could a one-dimensional transmission line with length $\gtrsim m_{a}^{-1}$.

Even for an experiment with all dimensions $\ll m_{a}^{-1}$, it is still possible to attain the sum-rule bound if we can enhance the EM energy fluctuations. ${ }^{16}$ Conceptually, the simplest way to accomplish this would be to use a material with high magnetic permeability $\mu$ [25]. Then, a magnetic field $B$ has EM energy density $B^{2} / 2$, but the magnetization $M=\left(1-\mu^{-1}\right) B$ contributes a negative energy density $-\left(1-\mu^{-1}\right) B^{2} / 2$, giving total energy density $B^{2} /(2 \mu)$. If the total magnetic energy (including the magnetization

\footnotetext{
${ }^{15}$ The proposal in [68] aims to avoid this suppression using a simple capacitive pickup. This relies on an alternative interpretation of axion electrodynamics, in which the axion is not necessarily derivatively coupled to SM fields $[69,70]$.

${ }^{16}$ This shows that one has to be careful in interpreting the results of papers such as [74,75], which show that the "induced EM fields" from the axion DM oscillation are suppressed, for background magnetic fields of small extent compared to the axion Compton wavelength. This is true, as defined, but does not have to lead to a smaller converted power, or worse detectability. For example, [74] makes the assumption that "Our detector is composed of a collection of time-independent charges and currents, $\rho_{e}$ and $J_{e}$," which automatically excludes e.g., resonant receivers.
} 


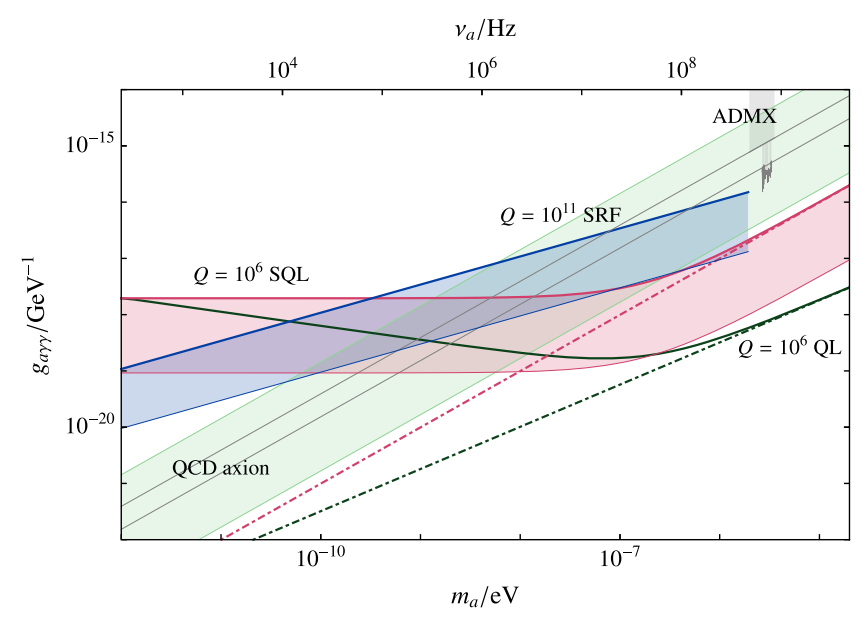

FIG. 1. Theoretical sensitivity limits for experiments searching

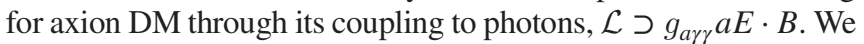
assume that axion DM has a virialized velocity distribution with $\delta v \sim 10^{-3}$, and density at Earth of $\rho_{a} \simeq 0.3 \mathrm{GeV} \mathrm{cm}^{-3}$. The red and dark green lines correspond to experiments using a static $4 \mathrm{~T}$ background magnetic field, with an experimental volume of $1 \mathrm{~m}^{3}$, and an integration time of one year per $e$-fold in axion mass. The blue lines correspond to up-conversion experiments, with a target frequency of $1 \mathrm{GHz}$, and taking a rms background magnetic field strength of $0.2 \mathrm{~T}$ in a volume of $1 \mathrm{~m}^{3}$ (with the same integration time assumptions). The $Q=10^{6} \mathrm{SQL}$ line (solid red) assumes a physical temperature of $10 \mathrm{mK}$, a quality factor of $10^{6}$, and SQLlimited op-amp readout (we choose an expected SNR value of 3 as our sensitivity threshold, in this and other cases). The solid red line assumes that the experiment operates in the quasistatic regime, with a consequent suppression in sensitivity (see Sec. IV C), while the dot-dashed line shows the full sum-rule-limited sensitivity (e.g., from using an appropriate matching circuit). The thin red line at the bottom of the red shaded region corresponds to the PQL-limited sensitivity (assuming quasistatic suppression); for higher quality factors, SQL-limited readout can approach this. The $Q=10^{6} \mathrm{QL}$ line (solid green) takes the same assumptions as the SQL line, but with quantum-limited readout (see Sec. III D), while the green dotdashed line shows this without the quasistatic suppression. The $Q=10^{11}$ SRF line (solid blue) corresponds to the isolated-linearamplifier sensitivity for an up-conversion experiment with physical temperature $1.5 \mathrm{~K}$, and mode quality factor $10^{11}$ [14]. The thin blue line at the bottom of the blue shaded region shows the PQL-limited sensitivity for these parameters - again, for higher quality factors, isolated amplifier readout can approach this [14]. The green diagonal band corresponds to the "natural" range of $g_{a \gamma \gamma}$ values at each QCD axion mass; if we write $g_{a \gamma \gamma}=\frac{\alpha_{\mathrm{EM}}}{2 \pi f_{a}}\left(\frac{E}{N}-1.92\right)$ [4], then the upper edge of the band is at $E / N=5[10]$, and the lower edge at $E / N=2$ [4]. The gray diagonal lines indicate the KimShifman-Vainshtein-Zakharov (upper, $E / N=0$ ) and Dine-Fischler-Srednicki-Zhitnitsky (lower, $E / N=8 / 3$ ) models. The gray shaded region corresponds to the parameter space excluded by ADMX [16,17].

term) of the mode has fluctuations $\sim \omega$, then the EM field contribution has fluctuations $\sim \mu \omega$, "borrowing" energy from the spins. If we make $\mu \sim 1 /(\omega L)^{2}$, we can attain

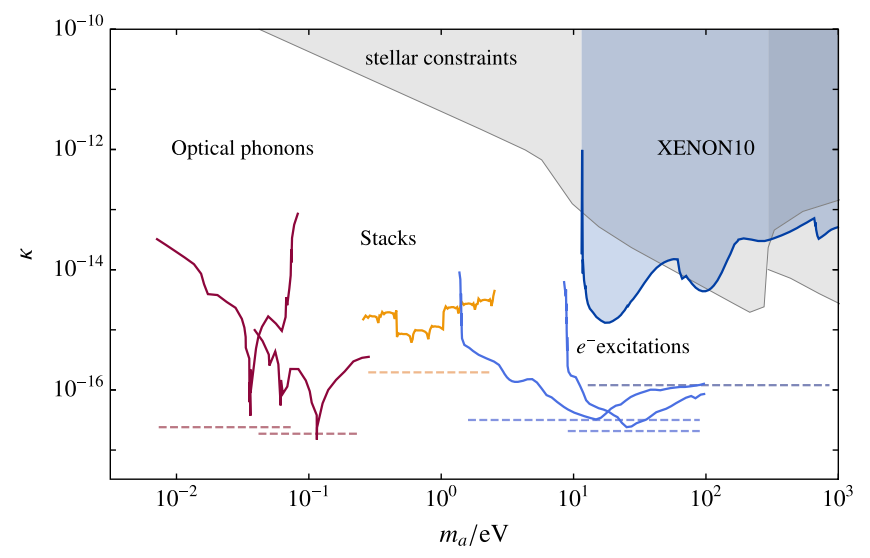

FIG. 2. Sensitivity projections for some existing and proposed dark photon DM detection experiments (solid lines), along with PQL sensitivity limits for the corresponding target volumes and integration times (dashed lines). See Sec. V for descriptions of the different projections. We assume that the dark photon DM has density at Earth of $\rho \simeq 0.3 \mathrm{GeV} \mathrm{cm}^{-3}$. The gray "stellar constraints" region corresponds to the bounds on energy loss from the Sun and from horizontal branch stars [71-73].

the sum-rule bound (at larger $\mu$, the wavelength $\lambda \sim \frac{2 \pi}{m \sqrt{\mu}}$ of the EM modes becomes comparable to $L$ [25]).

Circuit elements which can draw energy from e.g., a dc bias current, or a magnetic field bias, can also provide energy for the target mode's magnetic field to "borrow" from, enhancing its fluctuations. A toy example would be to connect an element with a negative inductance, such as a flux-biased loop [76], in series with the physical pickup inductor. In that case, the energy in the loop fluctuates downward, as the physical inductor's magnetic field energy fluctuates upward.

If such a setup has $\tilde{\chi}_{i} \geq 0$ for $\omega>0$, which must be true if it cannot on average emit power into the axion field, then it cannot beat the usual $\bar{P}$ bound of Eq. (74). For example, if we tried to use a negative inductor to cancel the inductance of the physical pickup loop to even higher precision than required to attain the $\bar{P}$ bound, then the energy of the $E_{F}$ field due to the changing magnetic field through the pickup loop would become important. The pickup loop necessarily stops behaving like an ideal inductance at some point, in analogy to the wavelength limit on enhancement from permeable materials. Similarly, if $\tilde{\chi}_{i}$ is well approximated, in the relevant frequency range, by a response function $\tilde{\chi}_{i}{ }^{\prime}$ that has $\tilde{\chi}_{i}{ }^{\prime} \geq 0$ for $\omega>0$, then the power absorbed is also bounded by Eq. (74).

The methods discussed above are interesting in principle, but would likely encounter practical difficulties. High-permeability materials, in particular, have major noise issues; they are generally lossy, have significant hysteresis and spin noise, and behave pathologically at high $(\gtrsim \mathrm{MHz})$ frequencies [25,77]. Approaches such as circuits with negative differential resistances (e.g., negative inductors) seem more promising, but may still have noise issues-for 
example, magnetic flux noise through flux-biased loops [77]. Whether setups with $\omega \tilde{\chi}_{i} \geq 0$, which are limited by sum rules, would be easier to implement than more general active approaches, is another interesting question. As discussed in the next subsection, a different approach to avoiding the quasistatic suppression at low axion masses is via up-conversion.

\section{Up-conversion}

Up-conversion experiments have been proposed at both microwave [31,33] and optical [26-28] frequencies. In the case of microwave cavities, it is simple to see that they can attain the sum-rule bounds from Sec. III E, to $\mathcal{O}(1)$. Suppose that we have a narrow-bandwidth axion signal, $a(t)=a_{0} \cos \left(m_{a} t\right)$. By equating $P_{\text {in }}=-\int d V E \cdot J^{(a)}$ to $P_{\text {loss }}=\frac{U \omega}{Q}$, we can obtain the formula from [31]

$$
P_{\text {sig }}=\frac{1}{8}\left(g a_{0}\right)^{2} m_{a}^{2} \frac{Q_{1}}{\omega_{1}} \frac{\left(\int d V E_{1} \cdot B_{0}\right)^{2}}{\int d V E_{1}^{2}}
$$

for the fully-rung-up, on-resonance signal power. Here, $B_{0}$ is the amplitude of the oscillating magnetic field, $B(t)=B_{0} \cos \left(\omega_{B} t\right)$. This gives a mass-averaged power of

$\bar{P} \simeq \frac{\pi}{8} g^{2} B_{0}^{2} \frac{\rho}{\Delta m} \frac{\left(\int d V E_{1} \cdot b_{0}\right)^{2}}{\int d V E_{1}^{2}} \equiv \frac{\pi}{8} g^{2} B_{0}^{2} \frac{\rho}{\Delta m} C_{01} V_{b_{0}}$.

Since $C_{01} \leq 1$, with equality if and only if $E_{1}$ and $B_{0}$ have the same profile, this agrees with Eq. (72). For example, the small-scale up-conversion experiment we discuss in [14], which drives the $\mathrm{TE}_{012}$ mode of a cylindrical cavity, and picks up signals in the $\mathrm{TM}_{013}$ mode, has $C_{01} \simeq 0.19$. The optical interferometry experiments proposed in [26-28] are phrased in somewhat different terms, but have the same parametric behavior, with the orthogonally polarized drive and signal modes having $C_{01} \simeq 1$.

For $m_{a} \gtrsim L^{-1}$, where static-field experiments would be outside the quasistatic regime, an up-conversion experiment with the same volume and rms magnetic field as a static-field experiment can have parametrically the same $\bar{P}$ value. However, as mentioned in Sec. I A, the achievable magnetic fields in up-conversion experiments will generally be smaller, being limited by both material properties and cooling systems. The driving required for the oscillating magnetic field will also introduce noise issues [14]. Furthermore, the cooling power required will generally restrict the attainable physical temperature to $\gtrsim 1 \mathrm{~K}$ [14]. Consequently, static-field experiments will generally be superior. A potential benefit of up-conversion experiments is that the magnetic field is entirely internal to the cavity, so superconducting cavities with very high quality factors can be used. For a static-field experiment, using an external magnetic field requires either a normal cavity, or a superconducting cavity in a vortex state [78], both of which have worse quality factors. However, this difference will generally not be enough to compensate for the disadvantages listed above.

For $m_{a} \lesssim L^{-1}$, static-field experiments are generically in the quasistatic regime, as discussed above, and have coupling sensitivity suppressed by $\sim(m L)^{-1}$. Up-conversion experiments do not suffer from this suppression, so they can scale better at axion frequencies $\ll \mathrm{GHz}$ (for lab-scale experiments). Figure 1 shows a quantitative version of this comparison. For the static-field experiments, we take nominal parameters of $V=1 \mathrm{~m}^{3}, B_{0}=4 \mathrm{~T}$, and $T=10 \mathrm{mK}$. For the nominal SRF experiment, we take $\sqrt{\overline{B_{0}^{2}}}=0.2 \mathrm{~T}$ (at the higher end of values that might be achievable with nioibium cavities [14]), $V=1 \mathrm{~m}^{3}$, and $T=1.5 \mathrm{~K}$ (as discussed in [14], cooling requirements make subkelvin temperature impractical). In a realistic experiment, there would likely be some form factor suppression (e.g., the $C \simeq$ $0.2^{2}$ estimate for static-field setups in [24]); Fig. 1 displays the volumewise limits, both so as to set a lower bound on the coupling sensitivity, and since our main point is to illustrate the different scalings. All of the curves use the single-pole sensitivity limits.

As investigated in [24,25], the SQL-limited sensitivity for searches in the quasistatic regime is constant as a function of axion mass. For $m_{a} \gtrsim T / Q_{1}$, the QL-limited coupling sensitivity is better, and scales $\propto m_{a}^{-1 / 4}$ in the quasistatic regime. Conversely, the up-conversion coupling sensitivity scales $\propto m_{a}^{1 / 2}$, so it is theoretically better at very low axion masses (of course, additional noise sources, e.g., vibrations, may make practical measurements difficult here [14]). Due to the smaller $B_{0}$ field, the SRF sensitivity is significantly worse at high $(\sim \mathrm{GHz})$ frequencies, where the quasistatic suppression is not significant. We also plot the static-field sensitivities without the quasistatic suppression (e.g., given a matching circuit of the kind discussed in the previous section), showing that, as expected, both the scaling and absolute sensitivity are superior to up-conversion in these circumstances. For measurement schemes which violate the assumptions of the different limits, e.g., quantum measurement approaches such as backaction evasion, different analyses would apply.

In our companion paper [14], we calculate basic sensitivity estimates for some specific SRF up-conversion setups, attempting to take into account some possible noise sources. Comparing these to the projections from staticfield experiments, we find that, with existing technologies, static-field experiments (even in the quasistatic regime) will most likely have better QCD axion reach. At very low axion masses, where the theoretical advantage of upconversion experiments is greatest, more careful investigation of noise sources would be required to understand whether improving on static-field experiments is plausible. Nevertheless, given the strong motivations for exploring axion parameter space, and the technological developments 
that may occur, it is important to understand the properties of different experimental approaches.

Comparing SRF setups to optical up-conversion experiments, the latter suffer from two major disadvantages compared to microwave frequencies. The first is that achievable electromagnetic field strengths at optical frequencies are much lower. Taking the optimistic parameters from [28], we can consider a $40 \mathrm{~m}$ long optical cavity with circulating optical power $\sim 1 \mathrm{MW}$ (for comparison, the circulating power in the LIGO interferometer arms is $\sim 100 \mathrm{~kW}$ [79]). This corresponds to a stored magnetic field energy of $\sim 0.1 \mathrm{~J}$. For comparison, the small-scale SRF experiment discussed in our companion paper [14] has stored energy $\sim \mathrm{kJ}$, while the nominal up-conversion experiment in Fig. 1 has $\sim 15 \mathrm{~kJ}$. The other issue is that, for an given signal power, the number of signal photons is much lower, by a factor $\sim \mathrm{eV} /(2 \pi \mathrm{GHz}) \sim 2 \times 10^{5}$. As a result, the theoretical sensitivity limits for optical experiments are significantly worse, as illustrated in Fig. 2 of [14]. Of course, optical experiments may be cheaper or simpler to implement than static-field or SRF experiments.

Microwave up-conversion experiments were also proposed in [32,33]. These have sensitivity projections which, at low axion masses, are significantly below our limits. As in the case of [68], it appears that this arises from an alternative interpretation of axion electrodynamics, in which the axion is not necessarily derivatively coupled to SM fields $[69,70]$.

\section{E. Down-conversion}

As discussed in Sec. III F, it is theoretically possible to increase $\bar{N}_{a}$ (and improve amplifier SNR) by using an oscillating $B_{0}$ field with frequency $\simeq m_{a}$, and concentrating the target mode's fluctuations at low frequencies. However, at frequencies $\omega_{s} \ll L^{-1}$, we are naturally in the quasistatic regime, where the fluctuations are suppressed by $\sim\left(\omega_{s} L\right)^{2}$. In that case, the down-conversion sensitivity is $\bar{N}_{a} \propto \omega_{s}$, and drops at lower frequencies. Hence, unless we circumvent the quasistatic regime, down-conversion experiments are only of interest for $\nu_{a} \gg \mathrm{GHz}$, where they could offer an idealized sensitivity up to $\sim \nu_{a} / \mathrm{GHz}$ times better than static-field experiments with the same $B_{0}$ amplitude. It is easy to think of toy implementations which can attain this bound. For example, if $\nu_{a}$ were in the optical regime, we could fill a microwave cavity with a photonic material, with periodicity at optical wavelengths. If a laser at the frequency of periodicity were shone through the cavity, then in the presence of an axion oscillation at almost the same frequency, the axions/laser photons would down-convert to microwave photons.

An obvious issue with these proposals is that achievable high-frequency $(\gg \mathrm{GHz})$ magnetic fields are orders of magnitude smaller than static ones, such that even an idealized advantage does not seem practical. Taking the optical-frequency $40 \mathrm{~m}$ cavity parameters from the previous subsection, the $\mathrm{eV} /(2 \pi \mathrm{GHz}) \sim 2 \times 10^{5}$ enhancement is not enough to compensate for the decreased magnetic field energy, compared to a nominal static-field experiment with $U_{B} \sim \mathrm{T}^{2} \times \mathrm{m}^{3} \sim$ MJ. Consequently, even with these extreme parameters, no conversion rate advantage would be achieved (even before considering the extra difficulties of detecting microwave photons vs optical photons).

\section{DARK PHOTON DM}

Apart from spin-0 candidates such as axions, light DM could also consist of oscillations of a vector field. Similar to spin-0 DM, there are a variety of possible nonthermal production mechanisms, including purely gravitational production from fluctuations "stretched" by inflation [43]. The simplest, and (at low vector masses) the least constrained, coupling of such a vector to the SM is the "kinetic mixing" coupling,

$\mathcal{L} \supset-\frac{1}{4} F_{\mu \nu} F^{\mu \nu}-\frac{1}{2} \kappa F_{\mu \nu} F^{\prime \mu \nu}-\frac{1}{4} F_{\mu \nu}^{\prime} F^{\prime \mu \nu}+\frac{1}{2} m^{2} A_{\mu}^{\prime} A^{\prime \mu}$

for a "dark photon" $A^{\prime}$. This is equivalent, after a field redefinition, to a massive vector with a small coupling to the EM current,

$\mathcal{L} \supset-\frac{1}{4} F_{\mu \nu} F^{\mu \nu}-\frac{1}{4} F_{\mu \nu}^{\prime} F^{\prime \mu \nu}+\frac{1}{2} m^{2} A_{\mu}^{\prime} A^{\prime \mu}-J_{\mathrm{EM}}^{\mu}\left(A_{\mu}+\kappa A_{\mu}^{\prime}\right)$.

The interaction term $\mathcal{L} \supset-\frac{1}{2} \kappa F_{\mu \nu} F^{\prime \mu \nu}=-\kappa\left(\partial_{\mu} A_{\nu}\right) F^{\prime \mu \nu}$ is equivalent, under integration by parts, to $\kappa A_{\nu} \partial_{\mu} F^{\prime \mu \nu}$. The Proca equation of motion for $A^{\prime}$ is $\partial_{\mu} F^{\prime \mu \nu}=m^{2} A^{\prime \nu}+\mathcal{O}(\kappa)$, so to leading order in $\kappa$, we can write the interaction term as $\mathcal{L} \supset \kappa m^{2} A_{\nu} A^{\prime \nu}$.

For a zero-velocity DM field, $A_{0}^{\prime}=0$, so the interaction term is $-\kappa m^{2} A \cdot A^{\prime}$. This corresponds to an effective current $J_{A^{\prime}}=-\kappa m^{2} A^{\prime}$, in analogy to the axion effective current $J^{(a)}=g \dot{a} B$. The dark photon's velocity dispersion means that the direction of $A^{\prime}$ is not constant, but changes over the field's coherence timescale, $\sim 10^{6} \mathrm{~m}^{-1}$. Hence, dark photon DM detection is analogous to axion DM detection, with an "effective magnetic field" that varies over the coherence timescale (and length scale) of the DM.

If we have a shielded experimental region, then the sensitivity to $\kappa$ is set by that region's volume. Unlike in the axion case, the frequency of the forcing term in the interaction Hamiltonian is set by the dark matter only, and does not depend on the target. Correspondingly, the upconversion and down-conversion scenarios do not apply. The limit on the absorbed power is simply 


$$
\bar{P} \lesssim \frac{\kappa^{2} \rho V m^{2}}{\Delta m} \frac{\pi}{2}
$$

giving

$$
\bar{N}_{A^{\prime}} \lesssim \kappa^{2} \rho V t \frac{m}{\Delta m} \frac{\pi}{2}
$$

Note that $\bar{N}_{A^{\prime}}$ does not vanish as $m \searrow 0$, unlike most rates involving dark photons, since the DM field amplitude becomes larger as $m$ becomes smaller (of course, once $t \lesssim m^{-1}$, the expression becomes invalid). Putting in representative numbers,

$$
N_{A^{\prime}} \lesssim\left(\frac{\kappa}{2 \times 10^{-19}}\right)^{2} \frac{\rho}{0.3 \mathrm{GeV} \mathrm{cm}^{-3}} \frac{V}{\mathrm{~m}^{3}} \frac{t}{\mathrm{yr}}
$$

for an order-one mass range. Consequently, for PQL or SQL-limited laboratory searches, $\kappa \sim$ few $\times 10^{-19}$ is the smallest kinetic mixing we could reasonably hope to detect. If $m \lesssim L^{-1}$, and we are in the quasistatic regime, then $\bar{N}_{A^{\prime}} \lesssim \kappa^{2} \rho_{A^{\prime}} V t(m L)^{2}{ }^{17}$

Almost any axion experiment using a roughly homogeneous, static background magnetic field will act as a dark photon detector "for free," even in the absence of the magnetic field. In particular, cavity/dielectric haloscopes can again be $\mathcal{O}(1)$ optimal for dark photon conversion; existing cavity haloscopes have set stringent limits on dark photon $\mathrm{DM}$ in the $\sim \mathrm{GHz}$ frequency range [81], while future proposals will often have sensitivity to dark photon DM significantly before they reach QCD axion sensitivity $[20,21,67]$. There are also many experiments and experimental proposals for which the addition of a strong background magnetic field would be practically difficult, so they can detect dark photons but not axions (e.g., experiments using superconducting phonon detectors, such as $[64,82])$.

In Sec. IVA, we noted how it is difficult to obtain efficient conversion from dielectric haloscopes at $\sim$ optical frequencies, due to a lack of suitable low-loss, high- $n$ dielectrics. These kinds of difficulties are generic - while achieving $\mathcal{O}(1)$ of the $\bar{P}$ limit is fairly simple at microwave frequencies, it is more challenging at higher DM masses. In contrast, detecting the converted excitations is often simpler, due to the reduced black body noise and more effective single-quantum detectors.

In Fig. 2, we show some sensitivity limits and projections for higher-mass dark photon DM detection

\footnotetext{
${ }^{17}$ This is true e.g., for a superconducting shield. If electric fields are screened, but low-frequency magnetic fields can penetrate, as is the case for a conducting cavity, then the field inside the shield will depend on the response of the material outside it to the DM field, potentially as far away as the DM coherence length. Experimental signatures of this effect for very low-frequency dark photon DM will be discussed in [80].
}

experiments, using different types of target excitations. We compare these to the PQL-limited sensitivities, for the appropriate target volumes and integration times. These illustrate the conversion efficiencies of the different targets and excitation types (all of the schemes assume efficient, almost background-free detection of converted excitations). This can help to identify how and where improved targets can be found, versus where larger volumes or longer integration times would be necessary to improve sensitivity.

At $m_{A^{\prime}} \gtrsim 10 \mathrm{eV}$, dark photon DM would have enough energy to ionize atoms, and such absorptions would be visible in WIMP (weakly interacting massive particle) detection experiments. Reference [83] analyzed the results of the Xenon10 experiment, and used these to place limits on dark photon DM. The dark photon absorption rate was calculated using the imaginary part of the photon propagator in liquid xenon [83]. As can be seen from the PQL bound, LXe is quite an inefficient dark photon absorber at these frequencies; the number of converted quanta is a factor of $\sim 7000$ lower than an ideal target occupying the same volume.

For slightly lower masses, dark photons can have enough energy to excite electrons in solid-state materials. Depending on the material, this excitation may be detected through ionization or scintillation signals [84]. In Fig. 2, we show background-free, $\mathrm{kg}$-yr exposure projections for sapphire and GaAs scintillators, from [82]. As the figure illustrates, these materials are much more efficient at converting dark photon DM, with conversion rates only a factor $\sim 5$ lower than ideal.

At lower frequencies, layered dielectrics have been proposed as a way to convert dark photon DM to photons [21], for detection using superconducting devices such as transition edge sensors (TESs) [85-90], nanowires [91], or microwave kinetic inductance detectors [92-94]. In Fig. 2, we show a nominal experimental projection using eight different 100-period stacks, each of area $\sim(18 \mathrm{~cm})^{2}$, covering a decade in dark photon mass range (each "chirped" stack can cover an $\sim 30 \%$ fractional mass range [21]). The material pairings used are $\mathrm{Si} / \mathrm{Al}_{2} \mathrm{O}_{3}, \mathrm{Si} / \mathrm{SiO}_{2}$, and $\mathrm{TiO}_{2} / \mathrm{SiO}_{2}$. These material choices give $\sim 1 / 30$ of the ideal conversion rate, corresponding to the $\frac{8}{\pi^{2}}\left(\frac{1}{n_{2}}-\frac{1}{n_{1}}\right)^{2}$ suppression from Sec. IV A, along with an $\sim 2 / 3$ misalignment factor from the dark photon polarization direction [21].

For even smaller dark photon masses, detectors with single-quantum sensitivity are challenging, but it is possible that superconducting technologies such as TESs or nanowires could achieve good enough energy resolution. Given such detectors, polar crystals have been proposed as a target for dark photon conversion [64,82]. Optical phonons in these crystals have gapped dispersion relations, allowing nonrelativistic dark photons to convert to lowmomentum optical phonons without the need for further momentum matching. One drawback of this approach is that the optical phonon dispersion relation is not easily 
tuned, so absorption is concentrated at the resonant frequency set by the optical phonon dispersion relation [with quality factor $\sim \mathcal{O}(100)$ [82]].

In Fig. 2, we show the sensitivity projections from [82] for $1 \mathrm{~kg}$-yr integrations with GaAs and sapphire crystals (assuming efficient and background-free detection). The resonant character of the absorption is clearly visible. Averaging across the mass range covered, the GaAs crystal converts $\sim 250$ fewer photons than an ideal target, while the sapphire crystal is only a factor $\sim 25$ below optimal.

\section{RELATIVISTIC ABSORPTION}

For nonrelativistic axion DM, we have seen that the maximum absorption rate averaged over some axion mass range scales with the magnetic field volume (multiplied by the rms magnetic field). While coherent absorption can be helpful in terms of absorbing into specific target modes (especially for background rejection purposes), it does not result in parametrically enhanced rates compared to incoherent absorption. For example, the ionization and electronexcitation techniques discussed in the previous section have the same scaling as dielectric haloscope absorption (while the former were discussed in the context of dark photon DM detection, we could turn them into axion experiments by placing them in a background magnetic field). However, for absorption of relativistic axions, coherent absorption can be parametrically advantageous.

In some situations, such as light-shining-through-wall experiments [95-100], axions are produced at a precise, known frequency. In this case, we want to concentrate the EM fluctuations in the receiver into a small frequency range. This can be accomplished using high-quality-factor coherent modes, or with e.g., narrow linewidth atomic transitions. Consequently, either "coherent" or "incoherent" absorption can be superior, depending on the linewidth achievable.

If the relativistic axion flux is spread over an $\mathcal{O}(1)$ range in frequencies, then the situation is different. Decomposing the EM field in the experimental volume into approximate plane wave modes, each has overlap with a range of axion momenta $\delta k \sim L^{-1}$. For axion masses small enough, this corresponds to a frequency range $\delta \omega \sim \delta k$, even for axion masses $\mathcal{O}(1)$ different from each other. If we can concentrate the EM field fluctuations for the appropriate mode into this frequency range, this enhances them by $\sim \omega / \delta \omega \sim \omega L$ over the broadband case, and so increases the absorption rate. In contrast, for a nonrelativistic axion DM signal, if we are $\mathcal{O}(1)$-uncertain as to $m_{a}$, then for each spatial mode we want to absorb into, the frequency uncertainty is $\mathcal{O}(1)$ (we will assume that the background magnetic field is static).

For an axion with relativistic momentum $k \gg m_{a}$, its frequency is

$$
\omega=\sqrt{k^{2}+m_{a}^{2}}=k\left(1+\frac{m_{a}^{2}}{2 k^{2}}+\ldots\right) .
$$

So, by varying the axion mass by $\mathcal{O}(1)$, we vary $\omega$ by $\sim m_{a}^{2} /\left(2 \omega^{2}\right)$. Consequently, we need $m_{a}^{2} \lesssim \omega / L$ to realize the full $\sim \omega L$ enhancement from above.

This enhancement for relativistic absorption is actually very simple to implement. If we know the approximate direction that the relativistic axions will be coming from, then we can construct a long tube facing in that direction, and fill it with an approximately uniform transverse magnetic field. This is exactly the setup of the CAST experiment [101], which looks for axions produced inside the Sun. If the axion mass is small enough such that $k_{a}-k_{\gamma}=k_{a}-\omega<L^{-1}$, i.e., $m_{a}^{2} /(2 \omega) \lesssim L^{-1}$, then axions traveling down the tube convert coherently to photons, with conversion probability $\propto(g B L)^{2}$, where $L$ is the length of the tube. Thus, if $F_{a} \sim a^{2} \omega$ is the axion flux, and $A$ is the tube's cross-sectional area, then the rate of converted photons is

$$
\Gamma_{\gamma} \sim F_{a} A(g B)^{2} L^{2} \sim(g a B)^{2}(A L)(\omega L) .
$$

Comparing this to Eq. (41), for a static-field experiment absorbing nonrelativistic axions over an $\mathcal{O}(1)$ mass range, we see that the relativistic case is enhanced by a factor $\sim \omega L$. This is just the number of wavelengths over which we can build up coherently, as expected. Photons in a mode with a specific momentum naturally have a specific frequency, realizing the concentration of the fluctuation spectrum that we wanted.

If $k_{a}-k_{\gamma}>L^{-1}$, then we would need to modify the photon dispersion relation inside the tube to obtain coherent conversion. For example, as done with CAST [102-105], we could introduce some gas into the tube, changing the refractive index for (x-ray) photons. In this case, the maximum possible enhancement, if $m_{a}$ is $\mathcal{O}(1)$ uncertain, is $\sim \omega^{2} / m_{a}^{2}$. For $\omega \sim m_{a}$, the enhancement disappears, as expected from our analysis of nonrelativistic DM.

If we allow the background magnetic field to oscillate in time, we could improve the theoretical detectability further by using the down-conversion ideas discussed above. However, the largest naturally occurring flux of relativistic axions is from the Sun, and is dominantly at $\sim \mathrm{keV}$ energies [106], so creating a suitable background field is not technologically plausible.

\section{A. Dark photons}

The case of relativistic dark photons is made more complicated by the different behavior of longitudinal and transverse excitations (for the nonrelativistic case, this difference is less important). For a plane wave traveling in the $z$ direction, the transverse polarization vectors for $A_{\mu}^{\prime}$ are spanned by $\epsilon_{\mu}^{(x)}=(0, \hat{x})$ and $\epsilon_{\mu}^{(y)}=(0, \hat{y})$, while the longitudinal polarization vector is $\epsilon_{\mu}^{(L)}=(-|k|, \omega \hat{z}) / m$ (for an on-shell excitation) [107]. For a vector coupling to a 
generic, nonconserved SM current, the $\sim \omega / m$ enhancement of the longitudinal polarization vector for relativistic excitations leads to energy-enhanced longitudinal emission [108]. Of course, the EM current that the dark photon couples to is conserved, so we instead expect the longitudinal mode to decouple as $m \rightarrow 0$; the $m^{2}$ factor in the coupling term $\mathcal{L} \supset \kappa m^{2} A^{\mu} A_{\mu}^{\prime}$ ensures that rates still decrease at least as fast as $m^{2}$ for $m \rightarrow 0$.

The maximum rate for the absorption of transverse $A^{\prime}$ is

$$
\Gamma \lesssim \begin{cases}\kappa^{2} \frac{m^{4}}{\omega^{4}} F V \omega(\omega L) & \text { for } m^{2} \lesssim \omega / L \\ \kappa^{2} \frac{m^{2}}{\omega^{2}} F V \omega & \text { for } \omega / L \lesssim m^{2} \lesssim \omega^{2}\end{cases}
$$

where $F$ is the dark photon flux, in analogy to the axion analysis above. Since SM photons are transversely polarized, it is simple to obtain the $(\omega L)$ and $\omega^{2} / \mathrm{m}^{2}$ enhancements corresponding to these cases. For $m^{2} \lesssim \omega / L$, the momentum difference between $A^{\prime}$ and free-space photons is small compared to the inverse size of the experiment, so a conducting cavity achieves this conversion rate (cf. the dark photon bounds from CAST [109]). To obtain a momentum match for $m^{2} \gtrsim \omega / L$, we would need to modify the dispersion relation of the photon inside the volume, e.g., using a gaseous or liquid medium, similar to CAST [102-105].

The maximum absorption rate for relativistic longitudinal dark photons is $\sim \omega^{2} / m^{2}$ times larger than the transverse rates above. However, to convert longitudinal $A^{\prime}$ to transversely polarized SM photons, we would need an anisotropic medium to set the photon polarization direction. If the medium is dense enough to accomplish this conversion efficiently, it will generally modify the dispersion relation of the SM photon as well. This would spoil the momentum match between the dark photon and the SM photon, and we would no longer get coherent conversion. While there are theoretically ways to get around thise.g., by multiplexing many wavelength-sized cavities together with the appropriate phase offsets-these seem difficult to implement at large enough $\omega$, so that the $\omega L$ coherence enhancement is significant for a laboratory-scale experiment.

If we do not take advantage of coherent conversion, then the maximum absorption rate for relativistic longitudinal dark photons is

$$
\Gamma \sim \kappa^{2} \frac{m^{2}}{\omega^{2}} F V \omega
$$

This is $\sim(\omega / L) / m^{2}$ times larger than the coherenceenhanced transverse rate for $m^{2} \lesssim \omega / L$, and equal to the transverse rate for $\omega / L \lesssim m^{2} \lesssim \omega^{2}$. Consequently, even without coherence enhancement, the theoretical limit on the absorption rate of longitudinal dark photons is always as

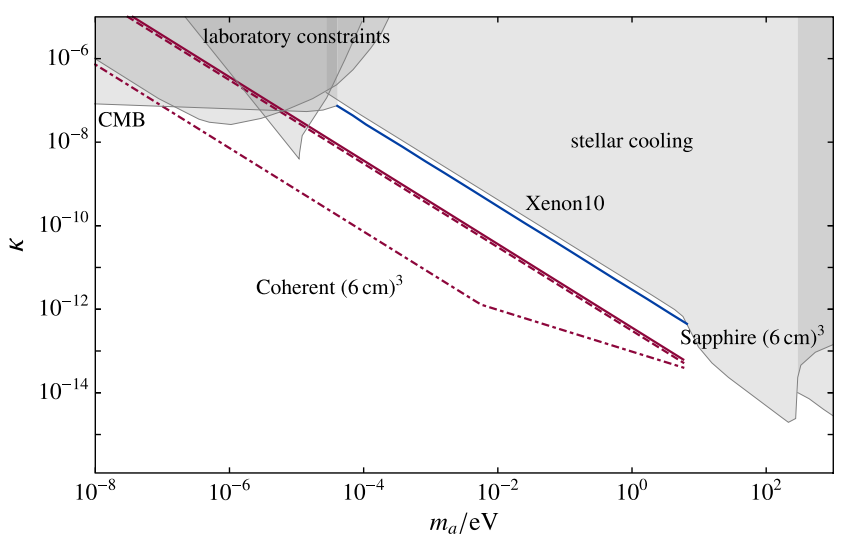

FIG. 3. Sensitivity of dark photon absorption experiments to the flux of longitudinal dark photons from the Sun. At $m \ll 300 \mathrm{eV}$, this flux is dominated by longitudinal emission [110], so we only consider absorption of longitudinal dark photons. The "Xenon10" line shows the limits from dark photon absorption in the Xenon10 experiment [73]. The solid red line shows the sensitivity for a sapphire target, with $1 \mathrm{~kg}-\mathrm{yr}$ exposure (as in Fig. 2). The dashed red line shows the constraints from an optimal incoherent absorber with the same volume $\left[\sim(6 \mathrm{~cm})^{3}\right]$, illustrating that sapphire is an almost optimal absorber, and in particular, much more efficient than liquid xenon [Xenon10 uses an $\sim(17 \mathrm{~cm})^{3}$ volume]. The dot-dashed line shows the maximum theoretical absorption rate for a cube of the same volume, taking advantage of coherent enhancement. As discussed in Sec. VI A, realizing this enhancement in a practical experiment would likely be very difficult. The gray regions correspond to existing constraints on dark photons from other observations [71-73, 98,111-114].

fast or faster than the coherence-enhanced absorption rate of an equivalent flux of transverse dark photons.

For $m$ much less than the plasma frequency in the Sun $\left(\omega_{p} \sim 300 \mathrm{eV}\right.$ in the solar core), the production rate of longitudinal $A^{\prime}$ from the Sun is $\propto m^{2}$, whereas the rate for transverse $A^{\prime}$ is $\propto m^{4}$. Consequently, for $m \ll 300 \mathrm{eV}$, the solar flux is dominated by longitudinal $A^{\prime}$ [110]. This means that longitudinal absorption is even more favored. Limits on this dark photon flux have been set by looking for longitudinal mode absorption in the Xenon10 detector [73]. However, the $A_{L}^{\prime}$ flux from the Sun peaks at $\omega \sim 10-100 \mathrm{eV}$, and as we saw in Sec. V, liquid xenon is a rather inefficient absorber of dark photons at these frequencies. If we used a more efficient absorption process, such as electronic excitations in sapphire, then significantly stronger constraints could be set. Figure 3 shows the projected constraints from dark photons from the Sun, using the same $1 \mathrm{~kg}$-yr sapphire experiment considered for DM detection in Fig. 2. The dashed line shows the theoretical sensitivity limit for incoherent absorption, using the same volume and integration time, illustrating that the sapphire is a close-to-optimal absorber. The dot-dashed line shows the additional sensitivity we could theoretically gain by taking advantage of coherent absorption of longitudinal 
$A^{\prime}$ (though we do not have a practical experimental proposal for this).

As well as the solar flux, there are other potential sources of relativistic dark photons, including dark radiation produced in the early Universe. If this has a temperature comparable to the cosmic microwave background, then most of its power is at $\sim \mathrm{mm}$ wavelengths, so there would be the potential for $\omega L \sim 10^{3}$ coherence enhancements in the converted power, for a meter-scale experiment. The polarization of the dark radiation would depend on the early Universe production mechanism, and would need to be considered in designing an experiment.

\section{CONCLUSIONS}

In this paper, we derived limits on the experimental detectability of axion DM, assuming that it couples to the SM through the $a F_{\mu \nu} \tilde{F}^{\mu \nu}$ operator. Similar analyses can be applied to other DM-SM couplings, most simply, to kinetically mixed dark photons, as we considered in Sec. V. For DM candidates which do not couple directly to the EM field, the EM sum-rule-based bounds we derived do not apply as straightforwardly. However, we can still use fluctuation-based (and/or energy absorption) analyses to bound the sensitivity of experimental searches.

In some cases, these can be related to the EM field fluctuations. For example, if DM consists of a vector which couples to the SM $B-L$ current, then its couplings to electrons and protons are the same as those of a dark photon with $\kappa_{\mathrm{eff}}=g_{B-L} / e$ (though its coupling to neutrons is different). So, in circumstances where neutrons are not important (for example, in dielectric haloscope detection schemes, where electrons dominate the dielectric response), the dark photon limits we derived will apply.

Analyzing the $B-L$ coupling directly, the PQL sensitivity is set by the fluctuations of the $B-L$ current in the target system. Since, for a shielded volume, the EM current fluctuations are related to the EM field fluctuations, andwhen neutrons are unimportant-the $B-L$ current is proportional to the EM current, the fluctuations of the $B-L$ current can be related to those of the EM field. For example, if $e$ were smaller, then the fluctuations of the electron number current in the shielding would have to be larger in order to cancel out the EM field fluctuations corresponding to the $\kappa_{\mathrm{eff}} \propto 1 / e$ behavior.

Of course, since there is not a perfect degeneracy between the effects of a $B-L$ vector and EM fields, the dark photon-type limits can be circumvented. In particular, one can look for the force exerted on neutron-containing objects, which can give good sensitivity at small DM masses [115].

A $B-L$ vector is an example of how there can be links between the EM field fluctuations, and the fluctuations of different operators. Such links may also be present in other cases, e.g., axionlike DM coupling to fermions, which for slow-moving fermions acts like an effective magnetic field.
We leave investigations of these and other examples to future work.

As discussed in Sec. VI, similar analyses to those in this paper can be applied beyond the scenario of DM searches, to other kinds of weakly coupled particle detection. In particular, quantum measurement theory has been extensively developed by the gravitational wave detection community (see e.g., [12]). Similar analyses can also be applied to DM scattering (rather than absorption) experiments, and to experiments looking for the production of new states.

Returning to the case of axion DM detection, our analyses clarify the limits on low-frequency axion detection, and motivate approaches for getting around the quasistatic suppression. In a companion paper [14], we perform a basic analysis of microwave up-conversion experiments in this regime, considering their sensitivity in light of plausible practical limitations. Alternatively, one could try to enhance the sensitivity of static-backgroundfield searches, via active or passive approaches. We leave investigations of such setups to further work. More generally, it would also be interesting to understand whether violating the $\omega \tilde{\chi}_{i}(\omega) \geq 0$ assumption, e.g., using unstable systems [116], could help improve sensitivity.

\section{ACKNOWLEDGMENTS}

We thank Masha Baryakhtar, Saptarshi Chaudhuri, Peter Graham, Junwu Huang, Kent Irwin, and Jesse Thaler for useful discussions. This research was supported in part by the National Science Foundation under Grant No. PHYS-1720397, the Gordon and Betty Moore Foundation Grant No. GBMF7946, and by the Munich Institute for Astro- and Particle Physics (MIAPP) which is funded by the Deutsche Forschungsgemeinschaft (DFG, German Research Foundation) under Germany's Excellence Strategy-EXC-2094-390783311.

\section{APPENDIX A: QUANTUM MEASUREMENT THEORY}

Almost all production mechanisms for light $(m \lesssim$ few eV) bosonic dark matter result in its state today being a coherent, classical-like oscillation, with high occupation number; for examples, see $[1-4,43]$. Since its couplings to a SM laboratory system are generally constrained to be very weak-in particular, far too weak for the interaction to significantly affect the DM state-it is valid to treat the DM as a fixed classical background field, for the purposes of detection. The question then becomes how well we can detect a small, classical forcing adding to the SM Hamiltonian. The ultimate limits on doing this are set by quantum mechanics, and are one of the subjects of quantum measurement theory [117]. Here, we give a brief overview of some basic results that we will use. 
Setting up the problem, suppose that we have a quantum system interacting with a classical oscillation $j(t)$ through the interaction Hamiltonian $H_{\text {int }}=g j(t) \hat{X}$, where $\hat{X}$ is some operator on the system, and $g$ is a coupling that we will take to be small. If we treat our quantum system in the interaction picture, and start with it in a state $|\psi\rangle$ at time $t_{0}$, then at leading order in $g$, by $t_{1}$ it has evolved to

$$
\left|\psi_{I}\left(t_{1}\right)\right\rangle \simeq\left(1-i g \int_{t_{0}}^{t_{1}} d t j(t) \hat{X}(t)\right)|\psi\rangle \equiv(1-i g \hat{V})|\psi\rangle .
$$

The overlap of $\left|\psi\left(t_{1}\right)\right\rangle$ with $|\psi\rangle$ is therefore

$$
\left|\left\langle\psi\left(t_{1}\right) \mid \psi\right\rangle\right| \simeq 1-\frac{1}{2} g^{2}\left\langle\psi\left|(\Delta \hat{V})^{2}\right| \psi\right\rangle
$$

where $\Delta \hat{V} \equiv \hat{V}-\langle\psi|\hat{V}| \psi\rangle$. Consequently, the ability to distinguish between the presence and absence of a weak signal is set by the fluctuations of the interaction Hamiltonian [118]. This is related to the theory of "quantum Cramer-Rao bounds" [39-41], and is sometimes referred to as an "energetic quantum limit" or "fundamental quantum limit" (FQL).

In our cases, the DM signal $j(t)$ will generally be narrow bandwidth, and it will be more useful to go to a spectral representation. Taking the same assumptions as above,

$$
\left|\left\langle\psi\left(t_{1}\right) \mid \psi\right\rangle\right| \simeq 1-g^{2} \int_{0}^{\infty} d \omega\left|\tilde{j}_{t}(\omega)\right|^{2} \bar{S}_{\Delta X \Delta X}(\omega)
$$

where $j_{t}(t)=j(t) \mathbf{1}_{t_{0}<t<t_{1}}$, and $\bar{S}_{\Delta X \Delta X}$ is the symmetrized spectral density of $\Delta X$. In the simplest case, where $j(t)$ is a single-frequency oscillation, $j(t)=j_{0} \cos (\omega t)$, we have

$$
\mathbb{P}_{\mathrm{ex}} \simeq \frac{1}{2} g^{2} j_{0}^{2} \bar{S}_{\Delta X \Delta X}(\omega) t_{\mathrm{exp}}
$$

where $t_{\text {exp }}=t_{1}-t_{0}$, and $\mathbb{P}_{\text {ex }} \equiv 1-\left|\left\langle\psi\left(t_{1}\right) \mid \psi\right\rangle\right|^{2}$ is the probability of changing the detector system's state. More generally, if we can treat $j(t)$ as a stochastic process, with some power spectral density $S_{j j}$, then in the limit where we evolve for a time long compared to the inverse bandwidth of spectral features in $S_{j j}$,

$$
\left\langle\mathbb{P}_{\mathrm{ex}}\right\rangle \simeq \frac{g^{2} t_{\mathrm{exp}}}{\pi} \int_{0}^{\infty} d \omega S_{j j}(\omega) \bar{S}_{\Delta X \Delta X}(\omega) .
$$

To gain some intuition for these results, it is helpful to consider the case where our system is a harmonic oscillator coupled to an external force, $\hat{X}=\hat{x}$. We will assume that the oscillator has some small coupling to other degrees of freedom, giving it a high quality factor $Q \gg 1$. In the ground state, we have

$$
\bar{S}_{x x}(\omega) \simeq \frac{Q}{1+Q^{2} \frac{\left(\omega^{2}-\omega_{0}^{2}\right)^{2}}{\omega_{0}^{4}}} \frac{1}{\omega_{0}} \frac{1}{M \omega_{0}}
$$

for $\omega$ close to $\omega_{0}$, where $\omega_{0}$ is the natural frequency of the oscillator, and $M$ is its mass. Here, $\frac{1}{M \omega_{0}}$ is the squared position uncertainty. By e.g., decreasing $M$ while keeping $\omega_{0}$ fixed, we increase the position uncertainty, and so decrease the momentum uncertainty. Since the external force changes the momentum of the system, having smaller momentum fluctuations helps to detect the forcing.

This illustrates how, for Gaussian states, the dependence on the fluctuations of $\hat{X}$ has a simple interpretation in terms of conjugate variables. However, things do not have to be that simple. For example, if the harmonic oscillator is initially in a number state, then $\left\langle n\left|\hat{x}^{2}\right| n\right\rangle=$ $\frac{2 n+1}{2 M \omega_{0}}$, and $\left\langle n\left|\hat{p}^{2}\right| n\right\rangle=\frac{M \omega_{0}}{2}(2 n+1)$-both the position and the momentum uncertainties are higher than for the ground state. However, by e.g., measuring the energy of the final state, we can still attain the $\bar{S}_{x x}$ bound [119]. Effectively, the rate for absorption (and emission) of quanta due to the forcing is Bose-enhanced by the initial occupation number. This provides an example of how, even for more complicated systems, the $\bar{S}_{\Delta X \Delta X}$ prescription still gives the correct answers.

In the discussion above, we assumed that the detector system was allowed to evolve for a time $t_{1}-t_{0}$, and only measured at the end. For many experimental setups, something closer to continuous monitoring is implemented; e.g., in resonant cavity experiments, the output port is connected to an amplifier. However, since we are only concerned with the fluctuations of $\Delta X$, as long as we include the rest of the system's dynamics in determining these (including measurements, feedback etc.), this does not present a problem (cf. the discussion of deferred measurement in [40]).

The above limits were based on knowing precisely which quantum state $|\psi\rangle$ our system starts in, and precisely how it would evolve from there. Other fluctuations, due to our uncertainty about the system's state (e.g., thermal fluctuations) have the opposite effect, making it harder to tell whether a signal is present. In some circumstances, $j(t)$ itself may be uncertain; for example, the Fourier components for a virialized DM signal are expected to have random amplitudes and phases. If these unknown Fourier amplitudes affect the system's response to the signal, then the effective SNRs for the independent components generally add in quadrature [120], rather than linearly, as per the Dicke radiometer formula [121].

\section{Linear amplifiers}

The FQL detectability limit discussed above applies to any type of detection system, as long as we properly calculate the quantum fluctuations of $\hat{X}$. However, in many 
cases, sensors are complicated nonequilibrium devices, and the fluctuations they cause may be difficult to compute. In addition, detection schemes may fail to obtain the FQL. Consequently, it is often helpful to consider the sensitivity limits for more restricted classes of sensors.

A common example of such a sensor, relevant to many axion detection experiments, is a linear amplifier. There is extensive literature on the quantum theory of linear amplifiers (see e.g., [35] for a review). In many circumstances, it is a good approximation to treat the fluctuations, both quantum and statistical, of measured and output quantities as Gaussian. Then, the relevant quantities can be summarized as "noise" spectral densities.

We will denote the PSD of backaction noise acting on $\hat{X}$ as $\bar{S}_{F F}(\omega)$, and the output imprecision noise (referred back to $X)$ as $\bar{S}_{X X}^{I}(\omega)$. The amplifier does not have to be connected "directly" to the $X$ degree of freedom for this description to make sense, so long as the whole system behaves linearly. A common setup is where a high-powergain amplifier is coupled weakly to the target system (where "weakly" means that it has a very subdominant effect on the system's damping). This is referred to as opamp mode [35]. In this scenario, the added noise associated with the amplification process is [35]

$\bar{S}_{X X}^{\mathrm{add}}(\omega)=|\tilde{\chi}(\omega)|^{2} \bar{S}_{F F}(\omega)+\bar{S}_{X X}^{I}(\omega)+2 \operatorname{Re}\left[\tilde{\chi}(\omega) \bar{S}_{X F}^{I}(\omega)\right]$

where $\bar{S}_{X F}^{I}$ denotes the correlation of the backaction noise, and the output imprecision noise referred back to $X$. The total output "noise" referred back to $X$ is $\bar{S}_{X X}^{\text {tot }}=\bar{S}_{X X}^{\mathrm{add}}+\bar{S}_{X X}^{n}+\bar{S}_{X X}^{\mathrm{ZPF}}$, where $\bar{S}_{X X}^{\mathrm{ZPF}}(\omega)=\tilde{\chi}_{i}(\omega)$ corresponds to the zero-point fluctuations of $X$, and $\bar{S}_{X X}^{n}$ summarizes the other noise contributions [e.g., for thermal noise, $\bar{S}_{X X}^{n}(\omega)=2 n_{T}(\omega) \tilde{\chi}_{i}$ ]. If we are attempting to detect a signal $j$, whose Fourier components have unknown phases, and integrate for a time long compared to inverse spectral bandwidths, then the expected SNR squared is

$$
\mathrm{SNR}^{2}=g^{4} t \int_{0}^{\infty} \frac{d \omega}{2 \pi}\left(\frac{\bar{S}_{j j}|\tilde{\chi}|^{2}}{\bar{S}_{X X}^{\text {tot }}}\right)^{2} .
$$

To maximize our SNR, we want to reduce $\bar{S}_{X X}^{\text {add }}$. For a high-gain amplifier, this is bounded below by $\bar{S}_{X X}^{\text {add }}(\omega) \geq$ $\left|\tilde{\chi}_{i}(\omega)\right|$ [35]. Achieving this "quantum limit" requires $\bar{S}_{I F}(\omega)=-\frac{\tilde{x}_{r}(\omega)}{2 \tilde{\chi}_{i}(\omega)}$, i.e., that the correlations between the imprecision and backaction noise are set by the phase of the response function.

In many circumstances, it is easier to implement linear amplifiers with uncorrelated imprecision and backaction noise. Following the gravitational wave detection literature [39], and papers such as [37], we will refer to this as the standard quantum limit (SQL), as opposed to the quantum limit (QL) in which correlations are permitted. Generally, it is the case that

$$
\bar{S}_{X X}^{I} \bar{S}_{F F}-\left|\bar{S}_{X F}^{I}\right|^{2} \geq \frac{1+\Delta\left[2 \bar{S}_{X F}^{I}\right]}{4}
$$

where $\Delta[z] \equiv\left(\left|1+z^{2}\right|-\left(1+|z|^{2}\right)\right) / 2$ [35]. This implies that $\bar{S}_{X X}^{I} \bar{S}_{F F} \geq \frac{1}{4}$. If there are no correlations, then $\bar{S}_{X X}^{\text {add }} \geq|\tilde{\chi}|^{2} \bar{S}_{F F}+\frac{1}{4 \bar{S}_{F F}}$, which is minimized by $\bar{S}_{F F}=\frac{1}{2|\tilde{\chi}|^{\prime}}$ giving $\bar{S}_{X X}^{\text {add }}(\omega) \geq|\tilde{\chi}(\omega)|$.

These limits apply to detection schemes which are invariant under time translation, usually referred to as "phase invariant" (i.e., they treat sine and cosine signals in the same way). By varying e.g., the detector coupling in a time-dependent way, sensitivity-enhancing schemes such as backaction evasion can be implemented [35].

The SNR limits look rather different from the fluctuation-based FQL limits discussed above. However, for equilibrium targets in the linear response regime, $\tilde{\chi}(\omega)$ is related to $\bar{S}_{\triangle X \Delta X}(\omega)$ via the fluctuation-dissipation relations. As we will see in the text, this can lead to closely related FQL and SNR bounds.

In some circumstances, we will want to go beyond the op-amp regime, and couple the detector more strongly to the target system. We discuss this in Sec. III C.

\section{APPENDIX B: ATOMIC AND MOLECULAR MAGNETIC FIELDS}

In the main text, the "background" magnetic field $B_{0}$ was generally taken to be a smoothed version, not taking into account the large magnetic fields inside molecules, atoms, nuclei, etc. This is justified since, if the electric field associated with signal excitations is slowly varying in space, then the interaction strength only depends on the magnetic multipole moments of subwavelength structures. For example, if we integrate over a volume containing some currents and spins, and the magnetic fields from sources outside the volume are small, then

$$
\int d V B=\frac{2}{3} m_{\mathrm{tot}}
$$

where $m_{\text {tot }}$ is the total magnetic dipole moment of the matter [122].

For the $\lesssim \mathrm{eV}$ excitations we considered, the smallest scale of spatial variation is e.g., motions of atoms in a lattice, or molecular vibrations. In particular, these are above the atomic scale. So, the magnetic field strength is, at best, that arising from atomic magnetic dipoles, which give an $\sim \mathrm{T}$ field. Thus, we cannot gain an advantage over using a strong, roughly uniform background magnetic field, which can be of multi-T strength. 
[1] M. Kawasaki, K. Saikawa, and T. Sekiguchi, Phys. Rev. D 91, 065014 (2015).

[2] A. Ringwald and K. Saikawa, Phys. Rev. D 93, 085031 (2016); Phys. Rev. D 94, 049908(E) (2016).

[3] R. T. Co, L. J. Hall, and K. Harigaya, Phys. Rev. Lett. 120, 211602 (2018).

[4] G. Grilli di Cortona, E. Hardy, J. Pardo Vega, and G. Villadoro, J. High Energy Phys. 01 (2016) 034.

[5] P. Agrawal, G. Marques-Tavares, and W. Xue, J. High Energy Phys. 03 (2018) 049.

[6] R. T. Co, E. Gonzalez, and K. Harigaya, J. High Energy Phys. 05 (2019) 162.

[7] R. T. Co, E. Gonzalez, and K. Harigaya, J. High Energy Phys. 05 (2019) 163.

[8] R. T. Co, L. J. Hall, and K. Harigaya, Phys. Rev. Lett. 124, 251802 (2020).

[9] P. W. Graham, I. G. Irastorza, S. K. Lamoreaux, A. Lindner, and K. A. van Bibber, Annu. Rev. Nucl. Part. Sci. 65, 485 (2015).

[10] L. Di Luzio, F. Mescia, and E. Nardi, Phys. Rev. Lett. 118, 031801 (2017).

[11] J. Jaeckel and A. Ringwald, Annu. Rev. Nucl. Part. Sci. 60, 405 (2010).

[12] H. Miao and Y. Chen, in Advanced Gravitational Wave Detectors, edited by D. G. Blair, L. Ju, C. Zhao, and E. J. Howell (Cambridge University Press, Cambridge, England, 2012), pp. 277-297.

[13] B. Pang and Y. Chen, Phys. Rev. D 99, 124016 (2019).

[14] R. Lasenby, Phys. Rev. D 102, 015008 (2020).

[15] P. Sikivie, Phys. Rev. Lett. 51, 1415 (1983).

[16] N. Du et al. (ADMX Collaboration), Phys. Rev. Lett. 120, 151301 (2018).

[17] T. Braine et al. (ADMX Collaboration), Phys. Rev. Lett. 124, 101303 (2020).

[18] L. Zhong et al. (HAYSTAC Collaboration), Phys. Rev. D 97, 092001 (2018).

[19] A. Droster and K. van Bibber (HAYSTAC Collaboration), in Proceedings of the 13th Conference on the Intersections of Particle and Nuclear Physics (CIPANP 2018) Palm Springs, California, USA, 2018 (2019), https://inspirehep .net/conferences/1655149.

[20] A. Caldwell, G. Dvali, B. Majorovits, A. Millar, G. Raffelt, J. Redondo, O. Reimann, F. Simon, and F. Steffen (MADMAX Working Group), Phys. Rev. Lett. 118, 091801 (2017).

[21] M. Baryakhtar, J. Huang, and R. Lasenby, Phys. Rev. D 98, 035006 (2018).

[22] P. Sikivie, N. Sullivan, and D. Tanner, Phys. Rev. Lett. 112, 131301 (2014).

[23] Y. Kahn, B. R. Safdi, and J. Thaler, Phys. Rev. Lett. 117, 141801 (2016).

[24] S. Chaudhuri, K. D. Irwin, P. W. Graham, and J. Mardon, arXiv:1904.05806.

[25] S. Chaudhuri, K. Irwin, P. W. Graham, and J. Mardon, arXiv:1803.01627.

[26] W. DeRocco and A. Hook, Phys. Rev. D 98, 035021 (2018).

[27] I. Obata, T. Fujita, and Y. Michimura, Phys. Rev. Lett. 121, 161301 (2018).

[28] H. Liu, B. D. Elwood, M. Evans, and J. Thaler, Phys. Rev. D 100, 023548 (2019).
[29] H. Padamsee, J. Knobloch, and T. Hays, RF Superconductivity for Accelerators, Wiley Series in Beam Physics and Accelerator Technology (Wiley, New York, 1998).

[30] A. Grassellino et al., Supercond. Sci. Technol. 30, 094004 (2017).

[31] P. Sikivie, arXiv:1009.0762.

[32] C. A. Thomson, B. T. McAllister, M. Goryachev, E. N. Ivanov, and M. E. Tobar, Phys. Rev. Lett. 126, 081803 (2021).

[33] M. Goryachev, B. Mcallister, and M. E. Tobar, Phys. Dark Universe 26, 100345 (2019).

[34] A. Berlin, R. T. D’Agnolo, S. A. Ellis, C. Nantista, J. Neilson, P. Schuster, S. Tantawi, N. Toro, and K. Zhou, J. High Energy Phys. 07 (2020) 088.

[35] A. A. Clerk, M. H. Devoret, S. M. Girvin, F. Marquardt, and R. J. Schoelkopf, Rev. Mod. Phys. 82, 1155 (2010).

[36] A. S. Chou, Astrophys. Space Sci. Proc. 56, 41 (2019).

[37] N. S. Kampel, R. W. Peterson, R. Fischer, P. L. Yu, K. Cicak, R. W. Simmonds, K. W. Lehnert, and C. A. Regal, Phys. Rev. X 7, 021008 (2017).

[38] B. J. Chapman, E. I. Rosenthal, J. Kerckhoff, B. A. Moores, L. R. Vale, J. Mates, G. C. Hilton, K. Lalumière, A. Blais, and K. Lehnert, Phys. Rev. X 7, 041043 (2017).

[39] B. Pang and Y. Chen, Phys. Rev. D 99, 124016 (2019).

[40] M. Tsang, H. M. Wiseman, and C. M. Caves, Phys. Rev. Lett. 106, 090401 (2011).

[41] H. Miao, R. X. Adhikari, Y. Ma, B. Pang, and Y. Chen, Phys. Rev. Lett. 119, 050801 (2017).

[42] The SQUID Handbook, edited by J. Clarke and A.I. Braginski (Wiley, New York, 2006).

[43] P. W. Graham, J. Mardon, and S. Rajendran, Phys. Rev. D 93, 103520 (2016).

[44] S. Weinberg, The Quantum Theory of Fields. Vol. 1: Foundations (Cambridge University Press, Cambridge, England, 2005).

[45] J. J. Sakurai, Modern Quantum Mechanics, revised ed. (Addison Wesley, Reading, MA, 1993).

[46] D. Tong, Lectures on kinetic theory, https://www.damtp .cam.ac.uk/user/tong/kintheory/kintheory.pdf (to be published).

[47] L. Necib, M. Lisanti, and V. Belokurov, Astrophys. J. 874, 3 (2019).

[48] P. Sikivie and J. R. Ipser, Phys. Lett. B 291, 288 (1992).

[49] K. Freese, P. Gondolo, H. J. Newberg, and M. Lewis, Phys. Rev. Lett. 92, 111301 (2004).

[50] L. D. Duffy and P. Sikivie, Phys. Rev. D 78, 063508 (2008).

[51] M. Vogelsberger and S. D. M. White, Mon. Not. R. Astron. Soc. 413, 1419 (2011).

[52] M. Fairbairn, D. J. Marsh, J. Quevillon, and S. Rozier, Phys. Rev. D 97, 083502 (2018).

[53] A. Arvanitaki, S. Dimopoulos, M. Galanis, L. Lehner, J. O. Thompson, and K. Van Tilburg, Phys. Rev. D 101, 083014 (2020).

[54] A. A. Clerk, F. Marquardt, and K. Jacobs, New J. Phys. 10, 095010 (2008).

[55] C. Boutan et al. (ADMX Collaboration), Phys. Rev. Lett. 121, 261302 (2018).

[56] B. M. Brubaker, Ph. D. thesis, Yale University, 2017. 
[57] C. Laflamme and A. A. Clerk, Phys. Rev. A 83, 033803 (2011).

[58] P. Brun et al. (MADMAX Collaboration), Eur. Phys. J. C 79, 186 (2019).

[59] J. Chiles (private communication).

[60] D. Horns, J. Jaeckel, A. Lindner, A. Lobanov, J. Redondo, and A. Ringwald, J. Cosmol. Astropart. Phys. 04 (2013) 016.

[61] M. Lawson, A. J. Millar, M. Pancaldi, E. Vitagliano, and F. Wilczek, Phys. Rev. Lett. 123, 141802 (2019).

[62] A. Arvanitaki, S. Dimopoulos, and K. Van Tilburg, Phys. Rev. X 8, 041001 (2018).

[63] E. J. Daw, Nucl. Instrum. Methods Phys. Res., Sect. A 921, 50 (2019).

[64] S. Knapen, T. Lin, M. Pyle, and K. M. Zurek, Phys. Lett. B 785, 386 (2018).

[65] R. Agnese et al. (SuperCDMS Collaboration), Phys. Rev. Lett. 121, 051301 (2018); 122, 069901(E) (2019).

[66] D. J. E. Marsh, K.-C. Fong, E. W. Lentz, L. Smejkal, and M. N. Ali, Phys. Rev. Lett. 123, 121601 (2019).

[67] S. Chaudhuri, P. W. Graham, K. Irwin, J. Mardon, S. Rajendran, and Y. Zhao, Phys. Rev. D 92, 075012 (2015).

[68] B. T. McAllister, M. Goryachev, J. Bourhill, E. N. Ivanov, and M. E. Tobar, arXiv:1803.07755.

[69] M. E. Tobar, B. T. McAllister, and M. Goryachev, Phys. Dark Universe 26, 100339 (2019).

[70] M. E. Tobar, B. T. McAllister, and M. Goryachev, Phys. Rev. Applied 15, 014007 (2021).

[71] H. An, M. Pospelov, and J. Pradler, Phys. Lett. B 725, 190 (2013).

[72] N. Vinyoles, A. Serenelli, F. L. Villante, S. Basu, J. Redondo, and J. Isern, J. Cosmol. Astropart. Phys. 10 (2015) 015.

[73] H. An, M. Pospelov, and J. Pradler, Phys. Rev. Lett. 111, 041302 (2013).

[74] J. Ouellet and Z. Bogorad, Phys. Rev. D 99, 055010 (2019).

[75] M. Beutter, A. Pargner, T. Schwetz, and E. Todarello, J. Cosmol. Astropart. Phys. 02 (2019) 026.

[76] V. Semenov, G. Danilov, and D. Averin, IEEE Trans. Appl. Supercond. 13, 938 (2003).

[77] S. Chaudhuri (private communication).

[78] D. D. Gioacchino et al., IEEE Trans. Appl. Supercond. 29, 1 (2019).

[79] B. P. Abbott et al. (LIGO Scientific and Virgo Collaborations), Phys. Rev. Lett. 116, 131103 (2016).

[80] M. Fedderke, P. Graham, and S. Kalia (to be published).

[81] P. Arias, D. Cadamuro, M. Goodsell, J. Jaeckel, J. Redondo, and A. Ringwald, J. Cosmol. Astropart. Phys. 06 (2012) 013.

[82] S. Griffin, S. Knapen, T. Lin, and K. M. Zurek, Phys. Rev. D 98, 115034 (2018).

[83] H. An, M. Pospelov, J. Pradler, and A. Ritz, Phys. Lett. B 747, 331 (2015).

[84] I. M. Bloch, R. Essig, K. Tobioka, T. Volansky, and T.-T. Yu, J. High Energy Phys. 06 (2017) 087.

[85] J. Dreyling-Eschweiler (ALPS-II Collaboration), in Proceedings of the 10th Patras Workshop on Axions, WIMPs and WISPs (AXION-WIMP 2014), Geneva, Switzerland (Verlag Deutsches Elektronen-Synchrotron Hamburg, 2014), pp. 63-66.
[86] J. Dreyling-Eschweiler, N. Bastidon, B. Dbrich, D. Horns, F. Januschek, and A. Lindner, J. Mod. Opt. 62, 1132 (2015).

[87] B. Cabrera, R. M. Clarke, P. Colling, A. J. Miller, S. Nam, and R. W. Romani, Appl. Phys. Lett. 73, 735 (1998).

[88] B. S. Karasik, S. V. Pereverzev, A. Soibel, D. F. Santavicca, D. E. Prober, D. Olaya, and M. E. Gershenson, Appl. Phys. Lett. 101, 052601 (2012).

[89] A. E. Lita, A. J. Miller, and S. W. Nam, Opt. Express 16, 3032 (2008).

[90] N. Bastidon, D. Horns, and A. Lindner, arXiv:1509.02064.

[91] K. M. Rosfjord, J. K. W. Yang, E. A. Dauler, A. J. Kerman, V. Anant, B. M. Voronov, G. N. Gol'tsman, and K. K. Berggren, Opt. Express 14, 527 (2006).

[92] B. A. Mazin, in American Institute of Physics Conference Series, edited by B. Young, B. Cabrera, and A. Miller (American Institute of Physics, Melville, NY, USA, 2009), Vol. 1185, pp. 135-142.

[93] P. Day, H. Leduc, B. A Mazin, A. Vayonakis, and J. Zmuidzinas, Nature (London) 425, 817 (2003).

[94] J. Gao, M. R. Vissers, M. O. Sandberg, F. C. S. da Silva, S. W. Nam, D. P. Pappas, D. S. Wisbey, E. C. Langman, S. R. Meeker, B. A. Mazin, H. G. Leduc, J. Zmuidzinas, and K. D. Irwin, Appl. Phys. Lett. 101, 142602 (2012).

[95] P. Sikivie, D. B. Tanner, and K. van Bibber, Phys. Rev. Lett. 98, 172002 (2007).

[96] F. Caspers, J. Jaeckel, and A. Ringwald, J. Instrum., 4, P11013 (2009).

[97] R. Bhre et al., J. Instrum. 8, T09001 (2013).

[98] M. Betz, F. Caspers, M. Gasior, M. Thumm, and S. W. Rieger, Phys. Rev. D 88, 075014 (2013).

[99] R. Janish, V. Narayan, S. Rajendran, and P. Riggins, Phys. Rev. D 100, 015036 (2019).

[100] P. W. Graham, J. Mardon, S. Rajendran, and Y. Zhao, Phys. Rev. D 90, 075017 (2014).

[101] V. Anastassopoulos et al. (CAST Collaboration), Nat. Phys. 13, 584 (2017).

[102] E. Arik et al. (CAST Collaboration), J. Cosmol. Astropart. Phys. 02 (2009) 008.

[103] M. Arik et al. (CAST Collaboration), Phys. Rev. D 92, 021101 (2015).

[104] S. Aune et al. (CAST Collaboration), Phys. Rev. Lett. 107, 261302 (2011).

[105] M. Arik et al. (CAST Collaboration), Phys. Rev. Lett. 112, 091302 (2014).

[106] S. Andriamonje et al. (CAST Collaboration), J. Cosmol. Astropart. Phys. 04 (2007) 010.

[107] E. Hardy and R. Lasenby, J. High Energy Phys. 02 (2017) 033.

[108] J. A. Dror, R. Lasenby, and M. Pospelov, Phys. Rev. D 96, 075036 (2017).

[109] J. Redondo, J. Cosmol. Astropart. Phys. 07 (2008) 008.

[110] J. Redondo and G. Raffelt, J. Cosmol. Astropart. Phys. 08 (2013) 034.

[111] A. Mirizzi, J. Redondo, and G. Sigl, J. Cosmol. Astropart. Phys. 03 (2009) 026.

[112] J. Jaeckel, J. Redondo, and A. Ringwald, Phys. Rev. Lett. 101, 131801 (2008).

[113] D. F. Bartlett and S. Loegl, Phys. Rev. Lett. 61, 2285 (1988). 
[114] E. R. Williams, J. E. Faller, and H. A. Hill, Phys. Rev. Lett. 26, 721 (1971).

[115] P. W. Graham, D. E. Kaplan, J. Mardon, S. Rajendran, and W. A. Terrano, Phys. Rev. D 93, 075029 (2016).

[116] H. Miao, Y. Ma, C. Zhao, and Y. Chen, Phys. Rev. Lett. 115, 211104 (2015).

[117] H. M. Wiseman and G. J. Milburn, Quantum Measurement and Control (Cambridge University Press, Cambridge, England, 2009).
[118] V. B. Braginsky, M. L. Gorodetsky, F. Ya. Khalili, and K. S. Thorne, AIP Conf. Proc. 523, 180 (2000).

[119] C. M. Caves, K. S. Thorne, R. W. P. Drever, V. D. Sandberg, and M. Zimmermann, Rev. Mod. Phys. 52, 341 (1980).

[120] C. W. Helstrom, Inf. Control 10, 254 (1967).

[121] R. H. Dicke, Rev. Sci. Instrum. 17, 268 (1946).

[122] J. D. Jackson, Classical Electrodynamics, 3rd ed. (Wiley, New York, 1999). 